\title{
Variational quark mass expansion and the order parameters of chiral symmetry breaking
}

\author{
Jean-Loï Kneur* \\ CERN, Theoretical Physics Division, CH-1211 Geneva 23, Switzerland \\ (Received 5 September 1996; published 28 January 1998)
}

\begin{abstract}
We investigate in some detail a "variational mass" expansion approach, generalized from a similar construction developed in the Gross-Neveu model, to evaluate the basic order parameters of the dynamical breaking of the $S U(2)_{L} \times S U(2)_{R}$ and $S U(3)_{L} \times S U(3)_{R}$ chiral symmetries in QCD. The method starts with a reorganization of the ordinary perturbation theory with the addition of an arbitrary quark mass $m$. The new perturbative series can be summed to all orders thanks to renormalization group properties, with specific boundary conditions, and advocated analytic continuation in $m$ properties. In the approximation where the explicit breakdown of the chiral symmetries due to small current quark masses is neglected, we derive Ansätze for the dynamical contribution to the "constituent" masses $M_{q}$ of the $u, d, s$ quarks, the pion decay constant $F_{\pi}$, and the quark condensate $\langle\bar{q} q\rangle$ in terms of the basic QCD scale $\Lambda_{\overline{\mathrm{MS}}}$. Those Ansätze are then optimized, in a sense to be specified, and also explicit symmetry breaking mass terms can be consistently introduced in the framework. The values of $F_{\pi}$ and $M_{q}$ obtained are roughly in agreement with what is expected from other nonperturbative methods. In contrast we obtain quite a small value of $|\langle\bar{q} q\rangle|$ within our approach. The possible interpretation of the latter results is briefly discussed. [S0556-2821(98)02303-0]
\end{abstract}

PACS number(s): 12.38.Aw, 11.15.Bt, 11.30.Rd, 12.38.Lg

\section{INTRODUCTION}

Although QCD is certainly established as the correct theoretical framework underlying strong interaction physics, a still challenging issue is to derive the low-energy properties of the strongly interacting spectrum from QCD "first principles,', due to our limited present skill with nonperturbative techniques. At very low energy, where (ordinary) perturbation theory cannot be applied, chiral perturbation theory (ChPT) [1] or extended Nambu-Jona-Lasinio (ENJL) models $[2,3]$ give a consistent framework in terms of basic parameters that have to be fixed from the data. Yet the link between those effective parameters and the basic QCD ones, the gauge coupling and the quark masses, remains largely unsolved. To have, typically, a determination of the pion decay constant $F_{\pi}$ and other similar low-energy quantities directly in terms of the basic QCD coupling constant $\alpha_{S}$ is clearly a desirable task. Lattice simulations are perhaps at present the only systematic approach to such questions, and indeed provide, among many other things, a determination of $\alpha_{S}$ [4] and also evidence for dynamical chiral symmetry breakdown (CSB) [5]. Yet a fully consistent treatment of chiral symmetry on the lattice is still missing, and there are also inherent difficulties in dealing with truly dynamical "unquenched", quarks, light meson masses, and related problems.

In the present paper, we investigate a new method to implement CSB directly from the basic QCD degrees of freedom, the quarks and gluons. More precisely we shall explore

\footnotetext{
* On leave from Laboratoire de Physique Mathématique et Théorique, U.R.A. 768 du C.N.R.S., F34095 Montpellier Cedex 5, France.
}

how far the basic QCD Lagrangian can provide, in a selfconsistent way, at least the basic CSB parameters, namely, the formation of dynamical quark masses, quark condensates, and the pion decay constant, in the limit of vanishing Lagrangian (current) quark masses. Such a qualitative picture of CSB can be made more quantitative by applying a new "variational mass" approach, recently developed within the framework of the anharmonic oscillator [6] and the Gross-Neveu (GN) models [7,8].

Before developing our construction, let us remind the reader that there are in fact two main phenomena which are believed to emerge from nonperturbative dynamics (and which are not fully understood from first principles), namely, the confinement and CSB, respectively. Even if those are certainly intimately related in the full QCD dynamics, it is legitimate to consider, at least in a first approximation, those two issues separately. QCD is a very rich theory involving many different nonperturbative aspects, and therefore any new method or model can most likely deal with a selected or simplified aspect of the full dynamics. After all, as far as the gross features of the chiral dynamics are concerned (that is, the existence of light pions and consequences in the form of low-energy theorems [9]), a picture without confinement but with CSB would not look very much different from the true QCD one. On the other hand, there is much evidence that confinement forces do play a more major role in the formation of heavier hadrons [10]. This relatively neat separation of the relevant scales is indeed one of the basic assumptions underlying several successful approaches to chiral dynamics, like the above-mentioned ChPT, ENJL models, etc., which generally do not include confinement properties in their framework. Somewhat closer to the present investigation, similar assumptions are often made in many dynamical CSB models, dealing with various levels of approximations in the treatment of the Schwinger-Dyson equations for the Green 
functions relevant to chiral dynamics. ${ }^{1}$ In contrast, there exists a quite radically different attitude towards CSB in QCD, advocating that the responsible mechanism is most probably the nonperturbative effects due to the instanton vacuum [13] or even more directly related to confinement [14]. However, even if the instanton picture of CSB is on general grounds well motivated, and many fruitful ideas have been developed in that context, ${ }^{2}$ as far as we are aware there is at present no sufficiently rigorous or compelling evidence for it. In any event, it is certainly of interest to investigate quantitatively the "noninstantonic" contribution to CSB, and we hope that our method is a more consistent step in that direction.

Although the basic idea was already developed in Refs. $[7,8]$ (see also [15] for a first acquaintance in QCD), we shall reformulate here the construction in some details for selfcontainedness reasons, and to take into account some crucial differences between QCD and the GN model. In addition we introduce explicit symmetry breaking mass terms consistently in the framework, which as we shall see play an important role in the determination of $\langle\bar{q} q\rangle$.

The method thus starts by considering an arbitrary quark mass term $m$ added to the massless QCD Lagrangian, whose dependence is calculated in perturbation theory with an expansion parameter $x$, interpolating between the massive free theory, at $x=0$, and the relevant interacting theory, at $x=1$. This starting point is similar to the one developed a long time ago and implemented in various different forms in Refs. [16-27]. There, it was advocated that the convergence of conventional perturbation theory may be improved by such a variational procedure in which the separation of the action into "free" and "interaction" parts is made to depend on some set of auxiliary parameters. The results obtained by expanding to finite order in this redefined perturbation series are optimal in regions of the space of auxiliary parameters where they are least sensitive to these parameters. Indeed, such regions are those expected to best approximate the exact answer, where there should simply be no dependence on the auxiliary parameters. ${ }^{3}$ Moreover strong evidence recently appeared that this optimized perturbation theory may lead to a rigorously convergent series of approximations even in strong coupling cases. In particular, the convergence of this variational-like procedure has been rigorously established in the case of zero- and one-dimensional field theories [30].

An essential novelty, however, is that the construction in $[7,8]$ combines in a specific manner the renormalization group (RG) invariance of the theory together with analytic continuation in $m$ properties: This, at least in a certain approximation to be motivated, allows us to reach an infinite order of perturbation in the parameter $x$, therefore presumably optimal, provided it converges. This leads to a set of nonperturbative Ansätze for the relevant CSB quantities, as functions of the variational mass parameter $m$ and renormal-

\footnotetext{
${ }^{1}$ See, e.g., Ref. [11] and the textbook by Miransky [12] for reviews and original references.

${ }^{2}$ For a review on such issues and an extended collection of original references, we refer to the textbook by Shuryak [10].

${ }^{3}$ For variants of the optimized variational-perturbation theory ideas, not based on minimization, but leading in many fieldtheoretical applications to similar results, see also Refs. [28,29].
}

ization scheme (RS) dependence parameters as well, which can be studied for extrema and optimized. Quite essentially, our construction also provides a simple and consistent treatment of the renormalization, reconciling the variational approach with the inherent infinities of quantum field theory and the RG properties. A comparison of the variational calculations with the known exact results [31] for the mass gap in the $O(N)$ GN model [32] is very satisfactory [8], in view of the fact that for arbitrary $N$ the (only known at present) second order nonlogarithmic perturbative terms have been used in the optimization. For QCD, one might, however, argue that the truly nonperturbative effects are lying in the infrared domain, for which a complete nonperturbative treatment is clearly beyond the scope of the present framework. We shall see in fact that our RG invariant Ansätz can be viewed as an (optimized) perturbation theory around a nontrivial fixed point of the RG evolution solutions, which in that sense avoids having to treat the infrared problems inherent to nonperturbative QCD. ${ }^{4}$ The physical relevance of such a framework can be judged a posteriori, e.g., by comparing our estimates with the results from other nonperturbative methods (like the lattice simulations $[4,5]$ or the spectral sum rules approach [35] typically), but unfortunately there is at present no rigorous way of defining the intrinsic error and convergence properties of the method, at least in four dimensions.

The paper is organized as follows: In Sec. II, we explain and motivate in details the construction and derive the main Ansatz for the dynamical CSB contribution to the "constituent" quark mass, in the exact chiral limit. A dynamical quark mass in QCD is not, strictly speaking, an order parameter, but it is interesting for at least two reasons: First it appears as a direct generalization of the rather intuitive concept of a (fermion) mass gap originating from dynamical CSB, as illustrated in many simpler models. Second, as we shall see, the concept and known properties of a gaugeinvariant and infrared finite pole mass enters as a basic ingredient in the Ansatz, and plays also some role in the determination of the genuine order parameters $\langle\bar{q} q\rangle$ and $F_{\pi}$. Concisely, our construction will be shown to amount to (i) taking the pole mass, as expressed as a function of the Lagrangian (arbitrary) running mass parameter $m(\mu)$ in some appropriate renormalization scheme (RS), (ii) constructing an Ansatz as a (contour) integral over the analytically continued mass parameter $m(\mu)$, which can be shown to formally resum the reorganized $x$ series, with a specific contour and appropriate RS choices avoiding some singularities, and (iii) finally optimizing the result with respect to $m(\mu)$ and the RS arbitrariness.

In Sec. III the construction is generalized to the case of a composite operator of arbitrary mass dimension $n$, and applied to the determination of the pion decay constant $F_{\pi}$. In Sec. IV we apply the construction to derive from similar

\footnotetext{
${ }^{4}$ Quite recently, an interesting approach has been developed [33] to determine the same CSB quantities, by using the exact "renormalization group flow" formulation [34]. Because of the central role played by RG properties, our present approach may be considered as having some similarities in aim with the latter one, albeit being totally different in the techniques used.
} 
arguments an expression for $\langle\bar{q} q\rangle$, introducing for this purpose explicit symmetry breaking mass terms. A summary and conclusions are given in Sec. V. We have also collected in Appendix A the relevant material on renormalization group properties, which fixes our normalizations and some definitions, and in Appendix B the analytic continuation techniques used in the derivation of the main Ansätze. For completeness we also display in Appendix $\mathrm{C}$ the perturbative expressions which are used as basic ingredients in the different Ansätze.

\section{DYNAMICAL QUARK MASSES}

\section{A. Preliminaries: QCD Lagrangian with variational mass}

Let us start with the bare QCD Lagrangian (for $n_{f}$ quark fields $q_{i}$ of identical masses)

$$
L_{Q C D}=-\frac{1}{4} G_{a}^{\mu \nu} G_{\mu \nu}^{a}+\sum_{i=1}^{n_{f}} \bar{q}_{i}\left(i \gamma_{\mu} D^{\mu}-m_{0}\right) q_{i},
$$

where

$$
\begin{gathered}
G_{a}^{\mu \nu} \equiv \partial^{\mu} G_{a}^{\nu}-\partial^{\nu} G_{a}^{\mu}-g_{0} f^{a b c} G_{b}^{\mu} G_{c}^{\nu}, \\
D^{\mu} q_{i} \equiv \partial^{\mu} q_{i}+i g_{0} T_{a} G_{a}^{\mu} q_{i}
\end{gathered}
$$

are, respectively, the gluon field strength tensor and the covariant derivative, $a$ is the color index, $i$ is flavor index, $T_{a}$ the $S U(3)_{c}$ generator, and $m_{0}$ and $g_{0}$ the bare mass and gauge coupling constant, respectively. As is well known the QCD Lagrangian (2.1) possesses for $m_{0}=0$ the additional chiral symmetry $S U\left(n_{f}\right)_{L} \times S U\left(n_{f}\right)_{R} \times U(1)_{L} \times U(1)_{R}$, at the classical level. ${ }^{5}$ In what follows we only consider the $S U\left(n_{f}\right)_{L} \times S U\left(n_{f}\right)_{R}$ part of the chiral symmetry, and for $n_{f}$ $=2$ or $n_{f}=3$ as physically relevant applications.

Following the treatment of the GN model [8], let us add and subtract a (bare) mass term, $m_{0} \sum_{i=1}^{n_{f}} \bar{q}_{i} q_{i}$, to be treated as an interaction term, to the massless QCD Lagrangian. This is most conveniently done by introducing a new perturbation parameter $x$, according to

$$
\begin{aligned}
L_{Q C D} \rightarrow & L_{Q C D}^{\text {free }}\left(g_{0}=0, m_{0}=0\right)-m_{0} \sum_{i=1}^{n_{f}} \bar{q}_{i} q_{i} \\
& +L_{Q C D}^{i n t}\left(g_{0}^{2} \rightarrow x g_{0}^{2}\right)+x m_{0} \sum_{i=1}^{n_{f}} \bar{q}_{i} q_{i},
\end{aligned}
$$

where $L_{Q C D}^{i n t}$ designates the usual QCD interaction terms, i.e., those proportional to $g_{0}$ or $g_{0}^{2}$ in Eq. (2.1). This is formally equivalent to substitute, everywhere in Lagrangian (2.1),

$$
m_{0} \rightarrow m_{0}(1-x), \quad g_{0}^{2} \rightarrow g_{0}^{2} x,
$$

\footnotetext{
${ }^{5} \mathrm{We}$ do not consider in particular the effects due to the breakdown of the axial $U(1)$ symmetry associated with instanton phenomena [36].
}

and therefore in any perturbative (bare) quantity as well, calculated in terms of $m_{0}$ and $g_{0}{ }^{6}$. Since the massless Lagrangian is recovered for $x \rightarrow 1, m_{0}$ is to be considered as an arbitrary mass parameter after the substitution (2.4). One expects to optimize physical quantities with respect to $m_{0}$ at different, possibly arbitrary orders of the expansion parameter $x$, eventually approaching a stable limit, i.e., flattest with respect to $m_{0}$, at sufficiently high order in $x$.

One immediately encounters, however, a number of obstacles: First, and quite essentially, before accessing to any physical quantity of interest for such an optimization, the theory has to be renormalized, and there is an inevitable mismatch between the expansion in $x$, as introduced above, and the ordinary perturbative expansion as dictated by the necessary counterterms, which at first spoils the whole picture. Moreover, for any physical quantity of interest in QCD one only knows at present the first few terms of the ordinary perturbative expansion in $g^{2}$, and the computational effort required to perform a perturbative calculation in $x$ is a priori similar. Finally, even if arbitrary higher order perturbation terms were available, it is easy to convince oneself that, at any finite order in the $x$ expansion, one always recovers a trivial result in the limit $m \rightarrow 0$ (equivalently $x \rightarrow 1$ ), which is the limit we are ultimately interested in to identify a mass gap (and more generally to identify spontaneous chiral symmetry breakdown, since an explicit Lagrangian mass term breaks the axial vector Ward-Takahashi identities explicitly [12]).

All these problems can be circumvented at once, by advocating a certain resummation Ansatz [8], exploiting the RG properties of the theory, plus analytic continuation properties in the arbitrary mass parameter $m$, which defines a candidate mass gap $M(m) .^{7}$ With respect to ordinary perturbation theory this RG-invariant Ansatz is exact for the leading and next-to-leading dependence in $m$ only, but the crucial point is that it resums as well the (reorganized) perturbation series in $x$. Moreover, taking the limit $x \rightarrow 1$ in this resummed expression no longer gives a zero mass gap. The construction can be systematically improved order by order (at least in principle, whereas in practice we obviously limit ourselves to the presently known highest perturbative orders for the relevant quantities). This is nevertheless hoped to be a sufficiently good starting point for further numerical optimization studies with respect to the (arbitrary) parameter $m .{ }^{8}$ For simplicity, we shall first illustrate the method by restricting our-

\footnotetext{
${ }^{6}$ It is implicitly understood that the same coupling constant reparametrization, Eq. (2.4), also affects, e.g., the Faddeev-Popov terms necessary at the loop-level calculations, which we will not display explicitly. Note therefore that the substitution (2.4) does not spoil the gauge invariance.

${ }^{7} \mathrm{We}$ will introduce several different definitions of a quark mass. To avoid confusion, we designate by $m$ the Lagrangian "current", mass (or any mass trivially related to the latter), while $M$ (with possibly different indices) is used for dynamical mass candidates at different stages.

${ }^{8}$ Such a situation is somewhat similar to many other variational approaches, where one often starts with a trial estimate, which one expects to be reasonably close to the exact result, before performing a variational calculation.
} 
selves in the next section to the first order of RG dependence. Generalization to higher orders will be discussed in the next sections.

\section{B. First order renormalization group mass Ansatz}

To be meaningful our formalism should be applied to RGinvariant and gauge-invariant quantities, in the QCD context. Therefore a reasonable starting point is to consider the pole quark mass, which is gauge invariant [37] to arbitrary perturbative orders [38] and infrared finite [37]. Using dimensional regularization (with space-time dimension $D=4-\epsilon$ ), the one-loop expression for the quark mass, using the onshell condition $\boldsymbol{q}=m_{0}$, is easily obtained as

$$
\begin{aligned}
& m_{0}\left[1+3 C_{F} g_{0}^{2} \frac{\Gamma(\epsilon / 2)}{(4 \pi)^{2-\epsilon / 2}}\left(m_{0}\right)^{-\epsilon}\left(1+\frac{2}{3} \epsilon+O\left(\epsilon^{2}\right)\right)\right. \\
& \left.+O\left(g_{0}^{4}\right)\right],
\end{aligned}
$$

where $C_{F} \equiv\left(N_{c}^{2}-1\right) /\left(2 N_{c}\right)$ for $S U\left(N_{c}\right)$ color (i.e., $C_{F}$ $=4 / 3$ for $\mathrm{QCD})$ and $\Gamma(x)$ the Euler gamma function. For $\epsilon \rightarrow 0$, expression (2.5) leads to the (renormalized) pole mass expression

$$
M_{1}^{P} \simeq \bar{m}\left\{1+2 \frac{\overline{\alpha_{S}}}{\pi}\left[\frac{2}{3}-\ln \left(\frac{\bar{m}}{\bar{\mu}}\right)\right]+O\left(\alpha_{S}^{2}\right)\right\},
$$

as expressed in terms of the modified minimial subtraction scheme $\quad(\overline{\mathrm{MS}}) \quad$ mass $\quad \bar{m} \equiv m(\bar{\mu}) \quad\left(\bar{\mu} \equiv \mu \sqrt{4 \pi} e^{-\gamma_{E} / 2}, \quad \gamma_{E}\right.$ $\simeq 0.577216 \ldots$, where $\mu$ is the arbitrary scale introduced by dimensional regularization), and coupling $\bar{\alpha}_{S} \equiv g^{2}(\bar{\mu}) /(4 \pi)$. In Eq. (2.6) we used the $\overline{\mathrm{MS}}$ mass counterterm $Z_{m}$, defined as $m_{0} \equiv Z_{m} \bar{m}$ and given from Eq. (2.5) as $Z_{m}\left(\bar{g}^{2}\right)=1$ $-\bar{g}^{2} /\left(2 \pi^{2} \epsilon\right)+O\left(g^{4}\right)$.

Our construction starts by first observing that RG properties gives us more information on higher orders than what appears in Eqs. (2.5), (2.6): One-loop calculations actually provide the leading $1 / \epsilon$ dependence of $Z_{m}$ and of the counterterm for the coupling $Z_{g}$ to all orders, as is well known:

$$
\begin{aligned}
& Z_{m} \equiv m_{0} / m=\left(1+\frac{2 b_{0}}{\epsilon} \bar{g}^{2}\right)^{-\gamma_{0} / 2 b_{0}}, \\
& Z_{g} \equiv\left(g_{0}^{2} / g^{2}\right) \mu^{-\epsilon}=\left(1+\frac{2 b_{0}}{\epsilon} \bar{g}^{2}\right)^{-1},
\end{aligned}
$$

where $\gamma_{0}$ and $b_{0}$ are the first order RG coefficients for the anomalous mass dimension and beta function, respectively (see Appendix A for the normalization used).

Now expression (2.5) using Eq. (2.7) suggests the following form of a bare resummed mass expression (restricted at the moment to the first RG order):

$$
M_{1}=\frac{m_{0}}{\left[1-b_{0} \Gamma(\epsilon / 2)(4 \pi)^{\epsilon / 2} g_{0}^{2}\left(M_{1}\right)^{-\epsilon}\right]^{\gamma_{0} / 2 b_{0}}},
$$

in close analogy with the mass-gap formula of the GN model [8]. Equation (2.8) is obviously RG invariant since expressed only in terms of $m_{0}$ and $g_{0}$. Introducing renormalized quantities $\bar{m}, \bar{g}^{2}$ from Eqs. (2.7), (2.8) is in addition finite to all orders, as easily checked, thanks to the recursivity in $M_{1}$, and reads, explicitly,

$$
M_{1}=\frac{\bar{m}}{\left[1+2 b_{0} \bar{g}^{2} \ln \left(\frac{M_{1}}{\bar{\mu}}\right)\right]^{\gamma_{0} / 2 b_{0}}} .
$$

Equation (2.9) resums the leading log (LL) dependence in $\bar{m}$ of the usual pole mass but, evidently, does not give its correct next-to-leading dependence in $\bar{m}$ [or even any of the "purely perturbative" (nonlogarithmic) finite corrections, such as the 2/3 factor appearing already at first order in Eqs. (2.5) and (2.6)]. Actually, the renormalized form (2.9) can alternatively be rigorously derived as a particular boundary condition imposed on the usual RG running mass. Indeed, consider the general solution for the RG evolution of the running mass,

$$
m\left(\mu^{\prime}\right)=m(\mu) \exp \left\{-\int_{g(\mu)}^{g\left(\mu^{\prime}\right)} d g \frac{\gamma_{m}(g)}{\beta(g)}\right\}
$$

in terms of the effective coupling $g(\mu)$, whose RG evolution is given as $\mu d g(\mu) / d \mu \equiv \beta(g)$. Solving Eq. (2.10) with the "fixed point" boundary condition

$$
M \equiv m(M),
$$

one easily obtains (to first RG order)

$$
M_{1}=\frac{m(\mu)}{\left[1+2 b_{0} g^{2}(\mu) \ln \left(\frac{M_{1}}{\mu}\right)\right]^{\gamma_{0} / 2 b_{0}}},
$$

which is nothing but Eq. (2.9) for $\mu=\bar{\mu}$. In particular, the recursive form of Eq. (2.12) with respect to $M_{1}$ can only be obtained thanks to the specific boundary condition in Eq. (2.11). Note that, although Eq. (2.9) can thus be obtained solely from the condition (2.11) imposed on the "current" mass $m(\mu)$, it has the usual properties of a pole mass. ${ }^{9}$ This coincidence between the pole mass $M$ and the current mass $m(M)$, evaluated at the scale $\mu \equiv M$, is of course only an artifact of our crude approximation, neglecting at the moment the nonlogarithmic perturbative corrections. Although the first order expression (2.9) having those properties could be guessed directly, the advantage of the latter derivation is that it gives a sound basis and precise link with the renormalization group behavior. Indeed, as will be derived in the next section, one can still obtain an exact solution of Eq. (2.10) with the boundary condition (2.11) at the next order explicitly, which is by construction entirely consistent with the next-to-leading RG dependence, to all orders. We shall

\footnotetext{
${ }^{9}$ In particular, $M_{1}$ in Eq. (2.12) is scale invariant, in contrast with $m(\mu)$ : i.e., an arbitrary change in $\mu$ in the current mass $m(\mu)$ is compensated, to all orders, by the $\ln \mu$ dependence in the denominator of Eq. (2.12). Equation (2.12) is also clearly gauge invariant, as the pole mass should be.
} 
also include later on the nonlogarithmic perturbative corrections, necessary to make contact with the genuine pole mass and to define a more realistic "mass gap" Ansatz.

Now, the most important property of the simple minded expression (2.9), which is also shared by the more realistic Ansätze to be later derived, is that it is nonzero in the chiral limit, $\bar{m} \rightarrow 0$. To see it explicitly, first rewrite identically Eq. (2.9) as

$$
M_{1}\left[\ln \left(M_{1} / \Lambda_{\overline{\mathrm{MS}}}\right)\right]^{\gamma_{0} / 2 b_{0}}=\hat{m}
$$

where for convenience we simply introduced the RGinvariant scale $\Lambda_{\overline{\mathrm{MS}}}=\bar{\mu} e^{-1 / 2 b_{0} \bar{g}^{2}}$ (at first RG order) and the scale-invariant mass $\hat{m} \equiv \bar{m}\left(2 b_{0} \bar{g}^{2}\right)^{-\gamma_{0} / 2 b_{0}}$. For fixed $\bar{g}^{2}$, the chiral limit is $\hat{m} \rightarrow 0$ in Eq. (2.13). Now, Eq. (2.13) may be seen as a function $\hat{m}\left(M_{1}\right)$, and requiring its inverse $M_{1}(\hat{m})$ to be defined on the whole physical domain $0<\hat{m}$ $<\infty$, and to match the ordinary perturbative asymptotic behavior for $\hat{m} \rightarrow \infty$, implies $M_{1}(\hat{m} \rightarrow 0) \rightarrow \Lambda_{\overline{\mathrm{MS}}} \cdot{ }^{10}$ This property of Eq. (2.9) is in contrast with the "one-loop improved", expression of the mass [obtained by replacing $M_{1} \rightarrow \bar{m}$ in the denominator of Eq. (2.9)], which would only give $M_{1} \rightarrow 0$ for $\bar{m} \rightarrow 0$. In other words, while Eqs. (2.8),(2.9) are perturbatively compatible with the usual (one-loop) RG behavior of the current mass, a specific choice of boundary condition for the RG running has selected a nontrivial "vacuum," by changing the behavior of the whole perturbative series in the chiral limit $\bar{m} \rightarrow 0$. Alternatively, one may derive the result $M_{1}=\Lambda_{\overline{\mathrm{MS}}}$ in the chiral limit as follows. From Eq. (2.13), defining

$$
F=\ln \left[\frac{M_{1}}{\Lambda_{\overline{\mathrm{MS}}}}\right] \equiv \ln \left[\frac{\hat{m}}{\Lambda_{\overline{\mathrm{MS}}}}\right]-\frac{\gamma_{0}}{2 b_{0}} \ln F
$$

on the physical branch, $0<\hat{m}<\infty$, the latter relation can be inverted as

$$
e^{F} F^{\gamma_{0} / 2 b_{0}}=\frac{\hat{m}}{\Lambda_{\overline{\mathrm{MS}}}}
$$

Now it is a simple algebraic exercise to see that Eq. (2.15) gives $F(\hat{m})$ as an expansion in powers of $\left(\hat{m} / \Lambda_{\overline{\mathrm{MS}}}\right)^{\left(2 b_{0} / \gamma_{0}\right)}$, for small $\hat{m}$ :

\footnotetext{
${ }^{10}$ There is a priori another possible solution of Eq. (2.13) in the chiral limit: $M_{1} \rightarrow 0$ when $\hat{m} \rightarrow 0$. However, it is easily seen that $M_{1}(\hat{m})$ has branch points at $M_{1}=\Lambda_{\overline{\mathrm{MS}}}$ and $M_{1}=e^{-\gamma_{0} / 2 b_{0}} \Lambda_{\overline{\mathrm{MS}}}$. The branch giving the trivial solution is only defined for $0 \leqslant|\hat{m}|$ $\leqslant\left(\gamma_{0} / 2 b_{0}\right)^{\gamma_{0} / 2 b_{0}} e^{-\gamma_{0} / 2 b_{0}} \Lambda_{\overline{\mathrm{MS}}}<\Lambda_{\overline{\mathrm{MS}}}$, and is therefore not compatible with the asymptotic perturbative behavior of Eq. (2.9) for $\bar{m} \rightarrow \infty$.
}

$$
F(\hat{m} \rightarrow 0) \simeq\left(\frac{\hat{m}}{\Lambda_{\overline{\mathrm{MS}}}}\right)^{2 b_{0} / \gamma_{0}}\left[1-\frac{2 b_{0}}{\gamma_{0}}\left(\frac{\hat{m}}{\Lambda_{\overline{\mathrm{MS}}}}\right)^{2 b_{0} / \gamma_{0}}+\ldots\right]
$$

which together with Eq. (2.13), i.e., $M_{1}=\hat{m} F^{-\left(\gamma_{0} / 2 b_{0}\right)}$, immediately gives $M_{1} \rightarrow \Lambda_{\overline{\mathrm{MS}}}$ for $\hat{m} \rightarrow 0$. While the latter derivation may be unnecessarily sophisticated at first order, it has the advantage of being the one easily generalizable to higher order, where we shall also find a nonzero mass in the chiral limit, $M=$ const $\times \Lambda_{\overline{\mathrm{MS}}}$, but with a no longer trivial proportionality constant. More importantly, it is also the most convenient procedure to analyze the chiral limit once having established an expression analogous to Eq. (2.9), but obtained directly from the variational perturbative (bare) expansion [as defined by the substitution (2.4)], which we address next. Our aim is to obtain a variational "mass gap" where the nontrivial chiral limit property of Eq. (2.9) is preserved, while at the same time providing us with a systematically improvable Ansatz, in accordance with the "variationally improved perturbation" principle.

Let us thus proceed with the $x$ parameter expansion, performing in Eq. (2.8) the substitution (2.4). Unfortunately, as announced in the last section, it is easily checked that the resulting expression is no longer finite: After the introduction of the usual counterterms, Eq. (2.7), some of the divergent terms in the denominator of Eq. (2.8) no longer cancel, except for $x \rightarrow 1$, in which case one only obtains the trivial result $M_{1} \rightarrow 0$. This is not very suprising since the precise all-order cancellations of divergences in Eq. (2.8), resulting from the above RG properties, have no a priori reasons to be compatible with the peculiar modification of the basic Lagrangian mass and coupling as implied by the substitution (2.4). The way out is to resum the $x$-generated series, denoted by $M_{1}(x)$, in a different manner [8]: By analytical continuation in $x$ one can find an adequate integration contour, resumming exactly the series $M_{1}(x)$ in the $x \rightarrow 1$ limit. This is explained in full detail in Appendix B. The net result gives an Ansatz (still at the first RG order approximation)

$$
M_{1}=\frac{1}{2 i \pi} \oint \frac{d v}{v} e^{v} \frac{v m_{0}}{f_{0}(v)^{\gamma_{0} / 2 b_{0}}}
$$

where $v \equiv q(1-x)$ has been introduced as an appropriate change of variable to analyze the $x \rightarrow 1, q \rightarrow \infty$ limit of the $q^{\text {th }}$-order expansion of $M_{1}(x)$, the contour is counterclockwise around the negative real axis, and we have defined

$$
f_{0}(v) \equiv 1-b_{0} g_{0}^{2} \Gamma\left[\frac{\epsilon}{2}\right]\left(m_{0} v\right)^{-\epsilon}\left(f_{0}\right)^{\epsilon \gamma_{0} / 2 b_{0}}
$$

whose expression is directly dictated from the denominator of Eq. (2.8) (see Appendix B for details). The crucial point is that Eq. (2.17) is now finite to all orders, while giving a nonzero result. More precisely, after renormalization Eq. (2.17) becomes

$$
M_{1}=\frac{1}{2 i \pi} \oint d v e^{v} \frac{\bar{m}}{f(v)^{\gamma_{0} / 2 b_{0}}}
$$


where the renormalized function $f(v)$, related to $f_{0}(v)$ above as $^{11}$

$$
f_{0}(1)=Z_{g} f(1)
$$

satisfies the (finite) recursion formula

$$
f=1+2 b_{0} \bar{g}^{2} \ln \left(\frac{\bar{m} v}{\bar{\mu}} f^{-\gamma_{0} / 2 b_{0}}\right) .
$$

Introducing a last convenient change of variable,

$$
F \equiv \frac{f}{2 b_{0} \bar{g}_{(v=1)}^{2}}=\frac{1}{2 b_{0} g^{2}\left(M_{1}\right)}
$$

Eq. (2.19) takes the form

$$
M_{1}=\frac{\Lambda_{\overline{\mathrm{MS}}}}{2 i \pi} \oint d y e^{y / m^{\prime \prime}} \frac{1}{F^{\gamma_{0} / 2 b_{0}}}
$$

where

$$
m^{\prime \prime} \equiv\left(\bar{m} / \Lambda_{\overline{\mathrm{MS}}}\right)\left(2 b_{0} \bar{g}^{2}\right)^{-\gamma_{0} / 2 b_{0}}, \quad y \equiv m^{\prime \prime} v
$$

are just the conveniently rescaled, dimensionless (scaleinvariant) "mass" parameter and integration variable, respectively. After those different manipulations, it is easy to see that one can use Eq. (2.14) to express $F$ in Eq. (2.23) as an expansion for small $y$, using Eq. (2.16) with the substitution $\left(\hat{m} / \Lambda_{\overline{\mathrm{MS}}}\right) \rightarrow y$ :

$$
F(y \rightarrow 0) \simeq y^{2 b_{0} / \gamma_{0}}\left(1-\frac{2 b_{0}}{\gamma_{0}} y^{2 b_{0} / \gamma_{0}}+\mathcal{O}\left(y^{4 b_{0} / \gamma_{0}}\right)\right) .
$$

This is nothing but a manifestation of the dimensional transmutation mechanism in the $m \rightarrow 0$ limit of QCD, although in a rather unconventional form: The function $F$, defined in Eq. (2.22) as a function of the running coupling, becomes, for $m \rightarrow 0$, a function of the (rescaled) mass only, $y \equiv m^{\prime \prime} v$. The original expression depending on $\left\{\bar{g}^{2}, \bar{m}\right\}$ now depends on $\left\{\Lambda \overline{\mathrm{MS}}, m^{\prime \prime}\right\}$ (where $m^{\prime \prime}$ is arbitrary), and we are ultimately interested in the chiral limit $m^{\prime \prime} \rightarrow 0$. From Eq. (2.25) it is clear that Eq. (2.23) has a simple pole at $y \rightarrow 0$, with the residue giving the announced nontrivial solution $M_{1}$ $=\Lambda_{\overline{\mathrm{MS}}}$.

The previous construction therefore shows that summing the $x$ series for the variational (bare) expansion and renormalizing, obtaining the contour integral in Eq. (2.19), is equivalent to performing the following steps: (i) Take the renormalized RG solution of Eq. (2.10), $M(\bar{m})$, with the condition (2.11); (ii) perform in $M(\bar{m})$ the substitution $\bar{m} \rightarrow \bar{m} v$, integrating the resulting expression around the cut negative real axis, with a specific weight $\oint(d v / v) e^{v}$ as in

\footnotetext{
${ }^{11}$ The simple relation in Eq. (2.20), involving the counterterm $Z_{g}$ only, is an accident of the first RG order approximation. At higher orders the relation is much more involved [8].
}

Eq. (2.19). ${ }^{12}$ Having this well-defined integral transform we can work directly with renormalized quantities and define formally an Ansatz at a given order as an appropriate generalization of the integral in Eq. (2.19), provided, however, that one does not encounter extra singularities in $v$. For simplicity let us postpone the question of the possible existence of extra singularities until Sec. II D, where this potential problem is addressed in detail.

It is now straightforward to introduce the necessary nonlogarithmic perturbative corrections to the (purely RG) above results: This is consistently done as

$$
M_{1}^{P}\left(m^{\prime \prime}\right)=\frac{\Lambda_{\overline{\mathrm{MS}}}}{2 i \pi} \oint d y e^{y / m^{\prime \prime}} \frac{1}{F^{\gamma_{0} / 2 b_{0}}}\left[1+\frac{2}{3} \frac{\gamma_{0}}{2 b_{0} F}+\mathcal{O}\left(\frac{1}{F^{2}}\right)\right],
$$

without changing anything in the contour integral properties, except that Eq. (2.26) no longer has a simple pole behavior at $y \rightarrow 0$. Equation (2.26) is nothing but a specific integral over the pole mass: Namely, taking the integrant for $v=1$ and expanding for small $\bar{g}^{2}$ by using relations (2.21) and (2.22), one explicitly recovers the usual pole mass expression with first order nonlogarithmic perturbative correction.

One may now optimize Eq. (2.26) with respect to $m^{\prime \prime}$, by performing (numerically) the $y$ integral. In fact, numerical integration is not mandatory: Since what we are interested in is the behavior for $m^{\prime \prime} \rightarrow 0$, it is equivalent to look at the properties of Eq. (2.26) for $y \rightarrow 0$. An expansion of Eq. (2.26) near the origin is provided from Hankel's formula,

$$
\frac{1}{2 i \pi} \oint d y e^{y / m^{\prime \prime}} y^{\alpha}=\frac{\left(m^{\prime \prime}\right)^{1+\alpha}}{\Gamma[-\alpha]},
$$

where the different powers $\alpha$ resulting from the expansion at arbitrary order near the origin have the form $\alpha=-1$ $+p 2 b_{0} / \gamma_{0}$, with $p=-1,0, \ldots$ integer. ${ }^{13}$

We stress that $m^{\prime \prime}$ (equivalently, $\bar{m}$ ) is meant to be an arbitrary but implicitly small parameter: Even if we ultimately seek for optimal values $m_{o p t}^{\prime \prime}$ as best approximations to the limit $m^{\prime \prime} \rightarrow 0$, at intermediate steps $m^{\prime \prime} \neq 0$ (i.e., $\bar{m}$ $\neq 0$ ) implies that the axial vector current Ward-Takahashi identities are explicitly broken. Eventually a small, physically acceptable explicit breaking [such as the PCAC (partial conservation of axial vector current) hypothesis [9]] may be considered, where $m_{o p t}^{\prime \prime}\left(\bar{m}_{o p t}\right)$ could be tentatively inter-

\footnotetext{
${ }^{12}$ Actually at the next order, solving Eq. (2.10) and taking the integral afterwards is not strictly equivalent to the derivation starting from the bare expressions. However, one can still show [8] that the two derivations are simply related by a particular renormalization scheme change.

${ }^{13}$ Note that Eq. (2.26) with Eq. (2.27) has some similarities with a Laplace-Borel transform: Indeed the analytic continuation in $m$ is almost equivalent (for $m \rightarrow 0$ ) to a continuation in the coupling $g$. The precise connection and the link with the renormalon singularities [39] associated with the usual Borel transform will be discussed in more detail elsewhere [40]. We shall, however, come back in the next sections to the structure of singularities of the integral (2.26) encountered in our framework.
} 
preted as the actual physical quark masses. But that is largely in contradiction with the basic principle adopted here, according to which $m^{\prime \prime}$ itself has no physical meaning, whereas only the optimal value of $M_{1}^{P}\left(m^{\prime \prime}\right)$ in Eq. (2.26) has. Indeed, in the (ideal) situation where the optima would be really flat, $m^{\prime \prime}$ would obviously not be well determined. It is therefore much more preferable to find a mean, eventually an approximate one, to reach the exact limit $m^{\prime \prime} \rightarrow 0$. The chiral Ward identities are then recovered and we can define the mass gap strictly in the chiral symmetric limit. We shall see in Sec. IV how to implement consistently an explicit symmetry breaking, physical mass term, independent of the arbitrary variational mass parameter $m^{\prime \prime}$.

\section{Second order dynamical mass Ansatz}

At the next RG order, the previous qualitative picture remains essentially unchanged, except that the derivation is somewhat more involved. The solution of Eq. (2.10) with Eq. (2.11), using the two-loop RG coefficients [41,42] given in Appendix A, is

$$
M_{2}=\bar{m} f^{-\gamma_{0} / 2 b_{0}}\left[\frac{1+\frac{b_{1}}{b_{0}} \bar{g}^{2} f^{-1}}{1+\frac{b_{1}}{b_{0}} \bar{g}^{2}}\right]^{\gamma_{1} / 2 b_{1}-\gamma_{0} / 2 b_{0}}
$$

where $f \equiv \bar{g}^{2} / g^{2}\left(M_{2}\right)$ satisfies

$$
f=1+2 b_{0} \bar{g}^{2} \ln \frac{M_{2}}{\bar{\mu}}+\frac{b_{1}}{b_{0}} \bar{g}^{2} \ln \left[\frac{1+b_{1} / b_{0} \bar{g}^{2} f^{-1}}{1+b_{1} / b_{0} \bar{g}^{2}} f\right],
$$

[note in Eqs. (2.28),(2.29) the recursivity in both $f$ and $M_{2}$ ], and the nonlogarithmic perturbative corrections are easily included as ${ }^{14}$

$$
M_{2}^{P} \equiv M_{2}\left(1+\frac{2}{3} \gamma_{0} \frac{\bar{g}^{2}}{f}+\frac{K}{\left(4 \pi^{2}\right)^{2}} \frac{\bar{g}^{4}}{f^{2}}+\mathcal{O}\left(g^{6}\right)\right),
$$

where the complicated two-loop coefficient $K$ was calculated exactly in Ref. [43] and is given explicitly in Appendix C. Equation (2.30) defines the pole mass including two-loop nonlogarithmic corrections, and can be easily shown in addition to resum the leading and next-to-leading logarithmic dependence in $\bar{m}$ to all orders (see Appendix A). The contour integral generalization of Eq. (2.26) is obtained, as explained before, after making the substitution $\bar{m} \rightarrow \bar{m} v$ in Eqs. (2.28)(2.30), as

$$
\frac{M_{2}^{P}\left(m^{\prime \prime}, a\right)}{\Lambda_{\overline{\mathrm{MS}}}}=\frac{2^{-C} a}{2 i \pi} \oint d y \frac{e^{y / m^{\prime \prime}}}{F(y)^{A}[C+F(y)]^{B}}
$$

\footnotetext{
${ }^{14}$ In Eq. (2.29) the recursivity operates on $M_{2}$, which by definition only depends on the RG evolution, therefore to be distinguished from the (pole) mass expression $M_{2}^{P}$ in Eq. (2.30).
}

$$
\times\left(1+\frac{\mathcal{M}_{1}(a)}{F(y)}+\frac{\mathcal{M}_{2}(a)}{F(y)^{2}}\right),
$$

where $\Lambda_{\overline{\mathrm{MS}}}$ is now the RG-invariant basic scale at two-loop order, in $\overline{\mathrm{MS}}[44],{ }^{15}$

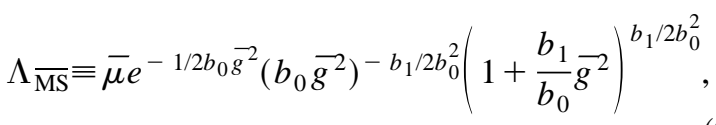

and the dimensionless (scale-invariant) arbitrary mass parameter reads

$$
m^{\prime \prime} \equiv\left(\frac{\bar{m}}{\Lambda_{\overline{\mathrm{MS}}}}\right) 2^{C}\left[2 b_{0} \bar{g}^{2}\right]^{-\gamma_{0} /\left(2 b_{0}\right)}\left[1+\frac{b_{1}}{b_{0}} \vec{g}^{2}\right]^{B} .
$$

In Eq. (2.31), $F$ now satisfies the recursive relation

$$
F(y) \equiv \ln [y]-A \ln [F(y)]-(B-C) \ln [C+F(y)],
$$

where $A, B, C$ are expressed in term of RG coefficients as

$$
A=\frac{\gamma_{1}}{2 b_{1}}, \quad B=\frac{\gamma_{0}}{2 b_{0}}-\frac{\gamma_{1}}{2 b_{1}}, \quad C=\frac{b_{1}}{2 b_{0}^{2}},
$$

explicitly given in Appendix A.

In Eq. (2.31) we have also introduced an extra parameter $a$, taking into account changes in the arbitrary renormalization scale, according to $\bar{\mu} \rightarrow a \bar{\mu}$. Indeed, even at the first RG order there are infinitely many ways of introducing the nonlogarithmic perturbative corrections to Eq. (2.23): For instance, we might have introduced the $\mathcal{O}(1 / F)$ terms via another definition of $F: F^{\prime}=F[1+\mathcal{O}(1 / F)]$ in place of Eq. (2.14), up to second order terms. This nonuniqueness is basically what is parametrized by $a$, and accordingly the perturbative coefficients $\mathcal{M}_{i}$ in Eq. (2.31) have a logarithmic dependence in $a$, simply dictated order by order from the requirement that Eq. (2.31) only differ from the original $\overline{\mathrm{MS}}$ scheme expression by higher order terms:

$$
\begin{aligned}
& \mathcal{M}_{1}(a)=\frac{\gamma_{0}}{2 b_{0}}\left(\frac{2}{3}-\ln a\right), \\
& \mathcal{M}_{2}(a)= \frac{1}{\left(2 b_{0}\right)^{2}}\left[\frac{K}{\left(4 \pi^{2}\right)^{2}}+\gamma_{0}\left(\frac{\gamma_{0}}{2}+b_{0}\right) \ln ^{2} a\right. \\
&\left.-\left(\frac{4}{3} \gamma_{0} b_{0}-\frac{\gamma_{0}^{2}}{3}+\gamma_{1}\right) \ln a\right] .
\end{aligned}
$$

Since we shall, however, continue expression (2.31) to the (nonperturbative) region of $m^{\prime \prime} \rightarrow 0$, in the manner described in the next sections, the $a$ dependence will eventually exhibit nontrivial extrema, and it is thus sensible to optimize the

\footnotetext{
${ }^{15}$ With our prescritions one should keep in mind that $\Lambda_{\overline{\mathrm{MS}}}$ depends on $n_{f}$, as usual [45].
} 
result with respect to $a,{ }^{16}$ since the unknown exact result would not depend on the arbitrary renormalization scale $\mu$. Note that $a \neq 1$ corresponds to a change of renormalization scale only, while keeping the renormalization scheme fixed, i.e., $\bar{g}^{2}=g^{2}(\bar{\mu})$ (equivalently $\Lambda_{\overline{\mathrm{MS}}}$ ) and $\bar{m}=m(\bar{\mu})$ fixed (to $\overline{\mathrm{MS}})$. Actually the situation is slightly more complicated, since at second perturbative order there are other possible changes of renormalization prescriptions than a simple change of scale, which do affect expression (2.31) and should therefore be taken into account in principle. This turns out to be an important aspect of our analysis and will be addressed in details in the next section.

For small $y, F(y)$ from Eq. (2.34) has the expansion

$$
F(y \rightarrow 0) \simeq A\left(u-\frac{B}{C} u^{2}+\mathcal{O}\left(u^{3}\right)\right), \quad u \equiv A^{-1} C^{(B-C) / A} y^{1 / A},
$$

again implying that Eq. (2.31) would give a simple pole at $y \rightarrow 0$ for vanishing perturbative correction terms, $\mathcal{M}_{1}$ $=\mathcal{M}_{2}=0$, with residue $(2 C)^{-C}$. Note that all the results of Sec. II B are consistently recovered by taking $b_{1}=\gamma_{1}=0$ and neglecting the nonlogarithmic perturbative corrections of $\mathcal{O}\left(g^{4}\right)$ in the different expressions above, Eqs. (2.28)-(2.37).

It is still possible to generalize to the next RG order the previous derivation, since in QCD the three-loop coefficients $b_{2}, \gamma_{2}$ are known [46], although the construction becomes quite cumbersome. In fact such a generalization is not worth doing, since one can in fact choose a renormalization scheme in which $b_{2}$ and $\gamma_{2}$ are set to zero, as well as all higher order coefficients. This is examined in more detail in the next section.

\section{Renormalization scheme changes and generalized Ansatz}

As it turns out, the simple picture emerging from the last section is unfortunately questionable as one realizes that, strictly speaking, formula (2.31) has extra singularities in the $y$ plane, in addition to the cut on the negative real $y$ axis, implicit in its derivation and in Eq. (2.27). After all, this specific contour was suggested by the known properties of the GN model, and it is not surprising if the analytic continuation in $m$ properties is more complicated in QCD. To begin, as is clear from Eq. (2.31) there is an extra branch point at $F=-C=-b_{1} /\left(2 b_{0}^{2}\right)$, which was not present in the first order Ansatz (2.26), and simply corresponds to the first nontrivial fixed point of $\beta(g)$ located at $\bar{g}^{2}=-b_{0} / b_{1}$. Fortunately it is harmless in the present QCD case, since $b_{1}>0$ implies that it is located along the already cutted $F<0$ real axis. ${ }^{17}$

\footnotetext{
${ }^{16}$ This procedure indeed gave very good results [8] in the GN model, where in particular for low values of $N$ the optimal values $a_{o p t}$ are quite different from 1 .

${ }^{17}$ In the GN model case the similar cut was more troublesome, since $b_{1}^{G N}<0$, and we used a specific Padé approximant construction [8] avoiding the cut. In a different context, this nontrivial fixed point has also been studied recently [47] in connection with renormalon properties.
}

Less trivially, the zeros of $d y / d F$ also give from Eq. (2.34) extra branch cuts in the $y$ plane, starting at the (complex conjugate) points

$$
y_{\text {cut }}\left(\gamma_{1}\right)=e^{F\left(\gamma_{1}\right)} F^{A}\left(\gamma_{1}\right)\left[C+F\left(\gamma_{1}\right)\right]^{(B-C)},
$$

where

$$
F\left(\gamma_{1}\right)=\frac{1}{2}\left(-\frac{\gamma_{0}}{2 b_{0}}\right)\left[1 \pm\left(1-4 \frac{\gamma_{1}}{\gamma_{0}^{2}}\right)^{1 / 2}\right]
$$

However, as can be seen the actual position of those branch points does depend on the scheme via the second coefficient of the anomalous mass dimension, $\gamma_{1}$ [the precise RS dependence of the latter is given in Eqs. (A6), (A8) of Appendix $\mathrm{A}]$. In the original $\overline{\mathrm{MS}}$ scheme, $F\left(\gamma_{1}^{\overline{\mathrm{MS}}}\right)$ gives extra cuts starting at $\operatorname{Re}\left[y_{\text {cut }}\right] \simeq 0.34(0.24), \operatorname{Im}\left[y_{\text {cut }}\right] \simeq \pm 0.74( \pm 0.76)$ for $n_{f}=2\left(n_{f}=3\right)$, respectively. Note that when looking at the limit of interest, $y \simeq 0$, using the expansion in Eq. (2.38), one never sees those extra cuts: At any finite order the series expansion only has the cut on the negative real axis according to Eqs. (2.27) and (2.38). These singularities may after all be an artifact of our extrapolation to very small $\mathrm{m}^{\prime \prime}$ of a "perturbative" (although resummed) relation, Eq. (2.34). Anyhow, the point is that using the expansion near the origin is invalidated if there are extra singularities lying in the way with $\operatorname{Re}\left[y_{\text {cut }}\right]>0$, since it would lead to an ambiguity of $\mathcal{O}\left(\exp \left(\operatorname{Re}\left[y_{c u t}\right] / m^{\prime \prime}\right)\right)$ for $m^{\prime \prime} \rightarrow 0$, in the determination of the integral $(2.31) .^{18}$

The way out is thus clear: If there exists values of $\gamma_{1}$ which move those extra cuts away (or which are such that they start at $\operatorname{Re}\left[y_{\text {cut }}\right]=0$ ), the expansion around the origin in $y$ is legitimate. Defining

$$
\gamma_{1}^{\prime} \equiv \gamma_{1}+\Delta \gamma_{1},
$$

one easily finds that $\operatorname{Re}\left[y_{\text {cut }}\right] \simeq 0$ for $\Delta \gamma_{1} \simeq 0.00267$ (0.00437) for $n_{f}=3\left(n_{f}=2\right)$, respectively. ${ }^{19}$ Therefore we can adjust a correct $\gamma_{1}^{\prime}$ by performing (perturbative) RS changes. As is explicit from Eqs. (A6), (A8) of Appendix A, this can be done either by a first order change in $g \rightarrow g^{\prime}$ or $m \rightarrow m^{\prime}$ (or both). One thus considers the most general RS change (but restricted to the second perturbative order, which is sufficient for our purposes) and optimize with respect to

\footnotetext{
${ }^{18}$ This is quite similar to the renormalon ambiguities [39]. Although the usual renormalons are not explicitly seen here due to the appearance of a mass gap [i.e., within the mass gap Ansatz (2.31), by construction there is no integration over the Landau pole region], the way in which the singularities in $y$ appear, namely, in a resummed expression relating a "reference" scale $M_{d y n} \simeq \Lambda_{\overline{\mathrm{MS}}}$ to an infrared scale $m^{\prime \prime} \simeq 0$, may be viewed as reminiscent from the renormalons. An essential difference is that in the present case those singularities occur in the analytic continuation of the mass parameter rather than the coupling, and that it is possible to move those singularities away by an appropriate RS change, as is discussed below.

${ }^{19}$ Actually $\operatorname{Re}\left[y_{\text {cut }}\right]$ is a (semi)periodic function of $\Delta \gamma_{1}$. We only consider solutions of $\operatorname{Re}\left[y_{\text {cut }}\left(\Delta \gamma_{1}\right)\right] \simeq 0$ nearest to the original $\overline{\mathrm{MS}}$ value $\left(\Delta \gamma_{1} \equiv 0\right)$ for $n_{f}=2,3$.
} 
this new arbitrariness. (As a side remark, we mention that one would also have $\operatorname{Re}\left[y_{\text {cut }}\right]<0$ with $\gamma_{1} \equiv \gamma_{1}^{\overline{\mathrm{MS}}}$, for $n_{f}$ $\geqslant 5$.) Note that the removing of the unwanted singularities is only possible at the two-loop RG order Ansatz, Eq. (2.31), due to the first occurrence of RS arbitrariness at two-loop order via $\gamma_{1}$, in MS schemes. More precisely, for the first order Ansatz, Eq. (2.26), an extra singularity occurs now with $\operatorname{Re}\left[y_{\text {cut }}\right]=\operatorname{Re}\left[\exp \left(-\gamma_{0} / 2 b_{0}\right)\left(-\gamma_{0} / 2 b_{0}\right)^{\gamma_{0} / 2 b_{0}}\right]>0$, which accordingly cannot be removed by a RS change.

We obtain after simple algebra the generalized dynamical mass Ansatz in the new (primed) scheme, in terms of the arbitrary RS change parameters $A_{1}, A_{2}, B_{1}, B_{2}$ defined in Appendix A:

$$
\begin{aligned}
\frac{M_{2}^{P}\left(a, m^{\prime \prime \prime}\right)}{\Lambda_{\overline{\mathrm{MS}}}^{\prime}}= & \frac{2^{-C} a}{2 i \pi} \oint d y \frac{e^{y / m^{\prime \prime \prime}}}{\left[F^{\prime}(y)\right]^{A^{\prime}}\left[C+F^{\prime}(y)\right]^{B^{\prime}}} \\
& \times\left(1+\frac{\mathcal{M}_{1}^{\prime}(a)}{F^{\prime}(y)}+\frac{\mathcal{M}_{2}^{\prime}(a)}{F^{\prime 2}(y)}\right),
\end{aligned}
$$

with

$$
\begin{aligned}
\mathcal{M}_{1}^{\prime}\left(a, B_{1}\right) & =\mathcal{M}_{1}(a)-\frac{B_{1}}{2 b_{0}}, \\
\mathcal{M}_{2}^{\prime}\left(a, A_{1}, B_{1}, B_{2}\right)= & \mathcal{M}_{2}(a)-\left(A_{1}+B_{1}\right) \frac{\mathcal{M}_{1}^{\prime}(a)}{2 b_{0}} \\
& -\frac{\left(B_{2}-\gamma_{0} B_{1}\right)}{4 b_{0}^{2}},
\end{aligned}
$$

and

$$
\Lambda_{\overline{\mathrm{MS}}}^{\prime}=\exp \left[\frac{A_{1}}{2 b_{0}}\right] \Lambda_{\overline{\mathrm{MS}}}
$$

[where relation (2.44) is exact to all orders [48]] and $F^{\prime}$ and $m^{\prime \prime \prime}$ have specific expansions in terms of the original $\overline{\mathrm{MS}}$ quantities $F$ and $m^{\prime \prime}$ that we will not need explicitly here. We also impose a further RS choice

$$
b_{2}^{\prime} \equiv 0, \quad \gamma_{2}^{\prime} \equiv 0
$$

which, according to relations (A7),(A8) in Appendix A, fixes $A_{2}$ and $B_{2}$ uniquely in terms of $B_{1}$ and $\Delta \gamma_{1},{ }^{20}$ and guarantees that the definition of $\Lambda_{\overline{\mathrm{MS}}}$ in Eq. (2.32) is unaffected, apart obviously from Eq. (2.44). In what follows we express all results in terms of the original $\Lambda_{\overline{\mathrm{MS}}}$ scale.

\section{E. Padé approximants and numerical results}

It is worth emphasizing at this point that our purpose is not to find a particular RS choice, which would "best fit",

\footnotetext{
${ }^{20}$ Although, e.g., $A_{2}$ does not appear explicitly in Eq. (2.42), we nevertheless need a prescrition to fix it, since it appears at the second order general RS change.
}

the expected order-of-magnitude result for the (dynamical) constituent mass (or similarly for $F_{\pi}$ and $m\langle\bar{q} q\rangle$ considered in the next sections). Indeed, it is very likely that with so much RS freedom at our disposal, one could make Eq. (2.42) fit almost whatever values one wishes. In contrast, what we are seeking is the flattest region in the arbitrary RS parameter space, in the "principle of minimal sensitivity",21 (PMS) sense. One soon realizes, however, that our extension of the PMS defines a rather complicated optimization problem: One has in principle to find the flattest possible extrema of Eq. (2.42), in the three independent parameter space $\left\{m^{\prime \prime}, a, B_{1}\right\}$, where in addition $\Delta \gamma_{1}$ is constrained to give good analyticity behavior of Eq. (2.42).

Fortunately, one can study this problem within some approximations, which we believe are legitimate. As above explained the Ansatz in Eq. (2.31) [or Eq. (2.42)] is already optimal with respect to $m^{\prime \prime}$ at $m^{\prime \prime}=0$, by construction, for vanishing pertubative nonlogarithmic corrections $\mathcal{M}_{i}=0$, and in this case the optimal result is the simple pole residue. Because of the nonlogarithmic, purely perturbative corrections (which are at present only known to second order for the mass), this simple picture is lost, but accordingly one may assume that the resulting expansion for small $m^{\prime \prime}$ is "as close as possible"' to an optimum (as would be the case if the sequence of approximations obtained by considering increasing orders of the $x$ expansion could be proved to converge, like in one-dimensional models [30]). Accordingly, we will define the $m^{\prime \prime} \rightarrow 0$ limit of Eq. (2.42) by a relatively crude but standard approximation of those perturbative corrections, rather than performing a numerical optimization with respect to $m^{\prime \prime}$. Indeed, as discussed at the end of Sec. II B, it is in addition physically motivated to reach $m^{\prime \prime} \rightarrow 0$, since the axial vector current Ward-Takahashi identities are recovered and a nonzero result signals the spontaneous chiral symmetry breakdown.

The approximation we are looking for is certainly not unique: Given the Ansatz (2.42), one may construct different approximant forms, leading to a finite limit for $m^{\prime \prime} \rightarrow 0$. We shall demonstrate the feasibility of our program in the simplest realization: Since the resulting expression will anyhow be optimized with respect to the RS dependence (entering any such approximants via the RS dependence of $\mathcal{M}_{i}$ ), we assume that it largely takes into account this nonuniqueness due to higher order uncertainties, in the standard PMS sense. The latter assumption is also supported by the results in the GN model [8], where several different approximants were tried and compared with the known exact results.

Padé approximants are generally known to greatly improve perturbative results [49] and in most cases have the effect of a smoothening of the RS dependence. We shall consider the following Padé approximant for the purely perturbative part of the integrant in Eq. (2.42):

$$
\operatorname{Padé}(F) \equiv \frac{F+\lambda\left(a, B_{1}\right)}{F+\rho\left(a, B_{1}\right)} \underset{F \rightarrow 0}{\rightarrow} \frac{\lambda(a)}{\rho(a)},
$$

which by construction restitutes a simple pole for $F \rightarrow 0$ in

\footnotetext{
${ }^{21}$ For an extended discussion of the PMS motivations, we refer to the original Stevenson's paper [16].
} 




FIG. 1. Dynamical quark mass $M_{q} / \Lambda_{\overline{\mathrm{MS}}}$ contribution from expression (2.47) with $n_{f}=2$ versus the scale parameter $a$, for different values of $B_{1}$ near the optimal (plateau) region. The exact plateau corresponds to $B_{1}=0.12 . \Delta \gamma_{1}$ is fixed to 0.00437 , such that $\operatorname{Re}\left[y_{\text {cut }}\right] \simeq 0$.

Eq. (2.42). Matching its perturbative expansion for $F \rightarrow \infty$ to the one in Eq. (2.42) one obtains

$$
\begin{aligned}
M_{2}^{\text {Padé }}\left(a, \Delta \gamma_{1}, B 1\right)= & \Lambda_{\overline{\mathrm{MS}}}(2 C)^{-C} a \exp \left\{\frac{A_{1}}{2 b_{0}}\right\} \\
& \times\left[1-\frac{\mathcal{M}_{1}^{2}\left(a, \Delta \gamma_{1}, B_{1}\right)}{\mathcal{M}_{2}\left(a, \Delta \gamma_{1}, B_{1}\right)}\right] .
\end{aligned}
$$

When defining Eqs. (2.46) and (2.47) one should be careful not to introduce new poles in the $F>0(y>0)$ plane. This simply implies that there are some constraints on the possible values of $\left(a, B_{1}\right)$ which, if we are lucky, do not invalidate their optimal values obtained from minimization.

We have performed a rather systematic study of the possible extrema of the Padé approximant formula (2.47) for arbitrary $a, B_{1}$, with $\Delta \gamma_{1}$ fixed such that the extra cuts start at $\operatorname{Re}[y] \simeq 0$. We did find an optimal region with respect to $a$, in the sense that it minimizes the second derivative at the maximum. ${ }^{22}$ The region of the parameter space in the vicinity of that extremum is illustrated in Fig. 1, as function of $a$ for different values of $B_{1}$. The plateau region has been determined more accurately using a numerical steepest descent method, requiring a minimal curvature. (For instance for $n_{f}$ $=2$ it corresponds to $a_{o p t} \simeq 1.42, B_{1, o p t} \simeq 0.12$.) Our results are also summarized in Table I (see Sec. V). The optimal values of the parameters $a, B_{1}, \Delta \gamma_{1}$ are consistent with the further requirement that Eq. (2.46) have no poles at $F>0$, as it should. Explicitly we obtain

$$
M_{2}^{\text {Padé }}(\mathrm{opt}) \simeq 2.97 \Lambda_{\overline{\mathrm{MS}}}(2)\left[2.85 \Lambda_{\overline{\mathrm{MS}}}(3)\right],
$$

for $n_{f}=2\left(n_{f}=3\right)$, respectively.

We shall see in the next section that we can relate the pion decay constant $F_{\pi}$ to $\Lambda_{\overline{\mathrm{MS}}}(2)$ or $\Lambda_{\overline{\mathrm{MS}}}(3)$, from which one

\footnotetext{
${ }^{22}$ Note that the second derivative at the extrema points is a good quantitative estimate of the flatness criteria even in a multidimensional parameter space: The intrinsic curvature at an extremum of the hypersurface as defined from, e.g., the approximant (2.47) is proportional to the product of its second derivatives with respect to the different parameters.
}

may eliminate $\Lambda_{\overline{\mathrm{MS}}}$ to obtain an evaluation of $M_{2}^{\text {Padé }}$ in the chiral limit. Anticipating these determinations of $\Lambda_{\overline{\mathrm{MS}}}$, Eq. (2.48) with $F_{\pi} \simeq 92 \mathrm{GeV}$ gives (still in the pure chiral limit)

$$
M_{2}^{\text {Padé }}(\mathrm{opt}) \simeq 500(447) \mathrm{MeV}
$$

for $n_{f}=2\left(n_{f}=3\right)$.

Finally, for a useful comparison, we also give the value of Eq. (2.47) in the $\overline{\mathrm{MS}}$ scheme (which may be thus considered as optimized with respect to $m^{\prime \prime}$ but in a fixed RS):

$$
M_{2}^{\text {Padé }}(\overline{\mathrm{MS}}) \simeq 0.99 \Lambda_{\overline{\mathrm{MS}}}(2)\left[1.00 \Lambda_{\overline{\mathrm{MS}}}(3)\right]
$$

Note, however, that according to the previous discussion, the results in Eq. (2.50) are a priori plagued by an ambiguity associated with the poorly placed extra singularities in the original $\overline{\mathrm{MS}}$ scheme. This may, however, give a qualitative idea of the effects of the above optimization with respect to the RS choice.

\section{GENERALIZATION TO COMPOSITE OPERATORS: $F_{\pi} / \Lambda_{\overline{\mathrm{MS}}}$}

\section{A. RG-invariant Ansatz for a composite operator}

We shall now derive an Ansatz similar to Eqs. (2.31), (2.42) for the pion decay constant $F_{\pi}$. The main idea is to do perturbation theory around the same RG evolution solution with the nontrivial fixed point, as specified by the function $F$ in Eq. (2.34), with perturbative corrections specific to $F_{\pi}$ obviously. One should first identify $F_{\pi}$ from a relation where the previous construction can be best generalized. A formal definition which suits all our purposes is the wellknown low-energy expansion of the axial-vector-axial-vector two-point correlation function $[1,50]$

$$
i \int d^{4} q e^{i q \cdot x}\left\langle 0\left|T A_{\mu}^{i}(x) A_{\nu}^{k}(0)\right| 0\right\rangle \simeq \delta^{i k} g_{\mu \nu} F_{\pi}^{2}+\mathcal{O}\left(p_{\mu} p_{\nu}\right) .
$$

In Eq. (3.1) $T$ is the time-ordered product and $A_{\mu}^{i}$ the axial quark current, $A_{\mu}^{i} \equiv\left(\bar{q} \gamma_{\mu} \gamma_{5} \lambda^{i} q\right) / 2$, where the $\lambda^{i}$, s are GellMann $S U(3)$ matrices or Pauli matrices for $n_{f}=3, n_{f}=2$, respectively. The nonvanishing of expression (3.1) implies 
TABLE I. Optimized results at different orders of the perturbative (non-RG) corrections for $n_{f}=2$ and $n_{f}=3$ as a function of the basic QCD scale $\Lambda_{\overline{\mathrm{MS}}}$. The corresponding values of the RS parameters at optima are indicated, when relevant. For $M / \Lambda_{\overline{\mathrm{MS}}}$ and $F_{\pi} / \Lambda_{\overline{\mathrm{MS}}}$ the zero order corresponds to neglecting completely the nonlogarithmic corrections, and taking the simple pole resulting from (first order) RG dependence. For $\langle\bar{q} q\rangle^{1 / 3} / \Lambda_{\overline{\mathrm{MS}}}$ the "zero" order corresponds in fact to the (unoptimized) formula (4.9).

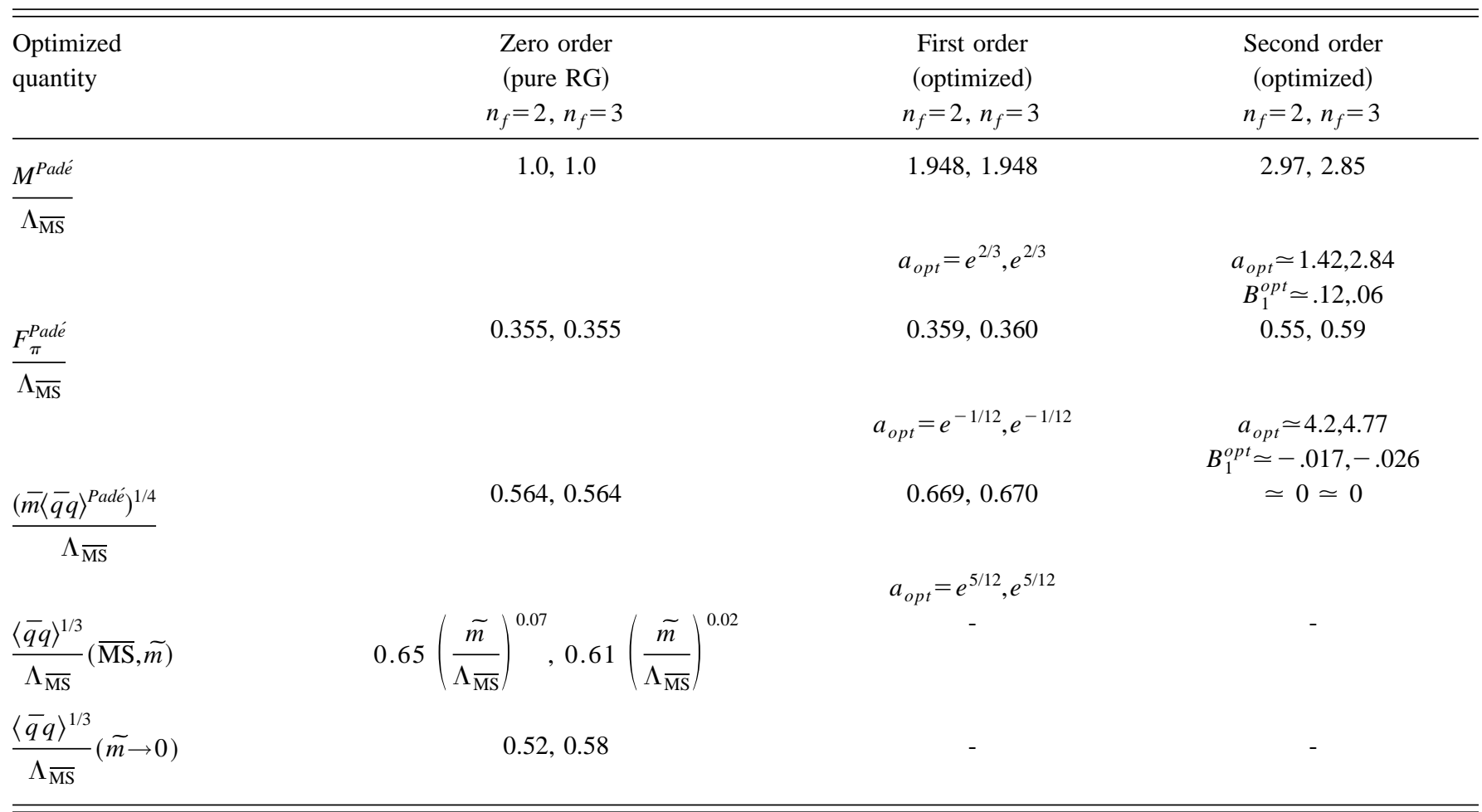

CSB: In other words $F_{\pi}$ is to be considered as an order parameter [51-53]. Since $F_{\pi}$ is expressed in terms of a gauge-invariant and RG-invariant composite operator in Eq. (3.1), one can apply a rather straightforward generalization of our Ansatz, exploiting in particular the RG resummation properties. The perturbative expression of Eq. (3.1) for $m$ $\neq 0$ is known to the three-loop order $[54,55]$, and is given explicitly in the $\overline{\mathrm{MS}}$ scheme in Appendix $\mathrm{C}$.

With a little bit of insight, a generalization of Eqs. (2.28)(2.30) to a composite operator $\mathcal{O}^{n}$ of naive mass dimension $n$ (depending only on $g$ and $m$ ) can be written as

$$
\mathcal{O}^{n} \simeq \frac{2 b_{0} m^{n}}{F^{n A-1}[C+F]^{n B}} \delta\left(1+\frac{\alpha}{F}+\frac{\beta}{F^{2}}+\cdots\right),
$$

in terms of $F$ as defined by Eq. (2.34) (for $y / m^{\prime \prime} \equiv v=1$ ), and where $\delta, \alpha, \beta$ are fixed by matching the perturbative expansion in a way to be specified next. In practice, we shall only consider Eq. (3.2) for the relevant cases of $n=2$ and $n=4$, corresponding to $F_{\pi}^{2}$ and $m\langle\bar{q} q\rangle$, respectively.

Formula (3.2) as it stands is not yet our final Ansatz and necessitates some comments. Apart from the trivial powers of $n$ dictated by dimensional analysis, a rather obvious difference with the mass formula is that the composite operator, not being a Lagrangian term, its perturbative expression starts at the one-loop, but zeroth order in $g^{2}$. To accommodate this fact with the correct $\mathrm{RG}$ properties, the expansion of Eq. (3.2) starts with a $1 / g^{2}$, due to the extra $2 b_{0} F$ factor. The $1 / g^{2}$ first order term anyhow cancels after a necessary subtraction which we discuss now. ${ }^{23}$

A more essential difference with the mass expression is that Eq. (3.1) is not finite even after mass and coupling constant renormalization. One can define a new, finite quantity after an adequate subtraction. In the present case, since our construction starts from perturbative expressions, this turns out to be nothing but the usual way of renormalizing a composite operator. Accordingly the prescription is (perturbatively) well defined [56] and unambiguous for a given RS choice. What is lost, however, is a part of the predictive power: Unlike the mass case, a consistent treatment of the subtracted terms (i.e., respecting RG invariance) implies that the unambiguous determination of the $1 / F^{n}$ perturbative terms in the final Ansatz necessitates information on the ( $n$ +1 ) order of perturbation theory.

\footnotetext{
${ }^{23}$ Note that the form and properties of Eq. (3.2) can alternatively be rigorously derived by following a construction starting from bare quantities, similar to the one explained in [8] for the vacuum energy of the GN model.
} 
In the two cases here considered, $F_{\pi}^{2}$ and $\bar{m}\langle\bar{q} q\rangle$ in the next section, this renormalization actually reduces to a simple subtraction of the operator $\left[m^{n} \times 1\right] .{ }^{24}$ This implies, from RG invariance, residual finite subtraction terms, affecting the perturbative correction terms $\alpha$ and $\beta$ in Eq. (3.2). Accordingly Eq. (3.2) is modifed to

$$
\begin{aligned}
\mathcal{O}^{n} \simeq & \frac{2 b_{0} \bar{m}^{n}}{F^{n A-1}[C+F]^{n B}} \delta\left(1+\frac{\alpha^{\prime}}{F}+\frac{\beta^{\prime}}{F^{2}}+\cdots\right) \\
& -\frac{\bar{m}^{n}}{\bar{g}^{2}} H\left(\bar{g}^{2}\right),
\end{aligned}
$$

where it is easily shown, using RG properties, that the finite subtraction function $H\left(\bar{g}^{2}\right) \equiv \sum_{i=0}^{\infty} H_{i} \bar{g}^{2 i}$ is determined perturbatively order by order (in the $\overline{\mathrm{MS}}$ scheme) from

$$
\left[n \gamma_{m}(\bar{g})+\frac{2}{\bar{g}} \beta(\bar{g})-\beta(\bar{g}) \frac{\partial}{\partial \bar{g}}\right] H\left(\bar{g}^{2}\right)=\frac{\bar{g}}{2} \frac{\partial c_{1}\left(\bar{g}^{2}\right)}{\partial \bar{g}} \text {. }
$$

In Eq. (3.4), $c_{1}\left(\vec{g}^{2}\right)$ is given by the residue of the $1 / \epsilon$ term in the perturbative series expansion for the relevant quantities, as given in Eqs. (C3) and (C10), respectively, for $F_{\pi}^{2}$ and $m\langle\bar{q} q\rangle$. The consistency of our formalism is checked by noting that the expansion of Eq. (3.3) in powers of $\bar{g}^{2}$ does reproduce correctly the LL and NLL dependence in $\bar{m}$ of the perturbative expansion of the composite operator to all orders, as well as the perturbative nonlogarithmic terms explicitly displayed in Eqs. (C3) and (C10).

\section{B. Generalized scheme Ansatz for $F_{\pi}$}

From the results of the last section one can now write an Ansatz for $F_{\pi}$, also taking into account the most general RS dependence to second order in a straightforward manner. After some algebra,

$$
\begin{aligned}
\frac{F_{\pi}^{2}}{\Lambda_{\overline{\mathrm{MS}}}^{2}}= & \exp \left[\frac{2 A_{1}}{2 b_{0}}\right] 2 b_{0} 2^{-2 C} \frac{a^{2}}{2 i \pi} \oint \frac{d y}{y} y^{2} e^{y / m^{\prime \prime \prime}} \\
& \times \frac{1}{F^{\prime 2 A^{\prime}-1}\left[C+F^{\prime}\right]^{2 B^{\prime}}} \\
& \times \delta_{\pi}\left(1+\frac{\alpha_{\pi}^{\prime}(a)}{F^{\prime}}+\frac{\beta_{\pi}^{\prime}(a)}{F^{\prime 2}}+\cdots\right),
\end{aligned}
$$

where

\footnotetext{
${ }^{24}$ In the general case [57], the renormalization of $\operatorname{dim}=4$ (gaugeinvariant) operators involves a mixing matrix for the operators $m\langle\bar{q} q\rangle, m^{4} \times 1$, and $G^{\mu \nu} G_{\mu \nu}$. However, the entry for $m\langle\bar{q} q\rangle$ only involves $m^{4} \times 1$ and $m\langle\bar{q} q\rangle$ itself [56]. As for $F_{\pi}^{2}$, the only gaugeinvariant operator of $\operatorname{dim}=2$ is $m^{2} \times 1$.
}

$$
\begin{aligned}
\delta_{\pi}= & \frac{N_{c}}{2 \pi^{2}} \frac{1}{\gamma_{0}-b_{0}}, \\
\alpha_{\pi}^{\prime}\left(a, \Delta \gamma_{1}\right)= & \frac{1}{2 b_{0}}\left[\frac{5}{6}\left(\gamma_{0}-b_{0}\right)-2 \pi^{2}\left(\gamma_{1}^{\prime}-b_{1}\right)\right. \\
& \left.-2\left(\gamma_{0}-b_{0}\right) \ln a\right], \\
\beta_{\pi}^{\prime}\left(a, \Delta \gamma_{1}, B_{1}\right)= & \frac{1}{\left(2 b_{0}\right)^{2}}\left[f_{\pi}^{(2)}\left(\Delta \gamma_{1}\right)+2 \gamma_{0}\left(\gamma_{0}-b_{0}\right) \ln ^{2} a\right. \\
+ & \left.\frac{\left(\gamma_{0}-b_{0}\right)}{6 \pi^{2}} \ln a\right]-\frac{2 B_{1}}{2 b_{0}} \alpha_{\pi}\left(a, \Delta \gamma_{1}\right) \\
+ & \frac{B_{1}}{\left(2 b_{0}\right)^{2}}\left(\frac{11}{3}\left(\gamma_{0}-b_{0}\right)-4 \pi^{2}\left(\gamma_{1}-b_{1}\right)\right),
\end{aligned}
$$

where $f_{\pi}^{(2)}$ is a complicated expression given explicitly in Appendix C.

An important point is that the subtraction function $H\left(\vec{g}^{2}\right)$ in Eq. (3.3) gives no extra contributions to the $y$ integral [8]: After introducing the contour integration as described in Sec. II and Appendix B, the purely perturbative subtraction gives an analytic function of $y$. Obviously, however, the actual values of the perturbative coefficients in Eqs. (3.7), (3.8) are affected by the subtraction. As previously mentioned, the unambiguous determination of $f_{\pi}^{(2)}$ in Eq. (3.8) involves, in a given (minimal) RS, knowledge of the three-loop coefficient of $1 / \epsilon$, obtained from the perturbative expression, Eq. (C3), of Appendix C. Note also that the dependence upon $\Delta \gamma_{1}$ in Eq. (3.7) is only through $\gamma_{1}^{\overline{\mathrm{MS}}} \rightarrow \gamma_{1}^{\prime}$, and the dependence on $B_{1}$ only appears in the second order term, $\beta_{\pi}^{\prime}$, in contrast with the mass case. As well there is no dependence upon $B_{2}$ or $A_{2}$ at this (second) order. All these properties are of course consequences of the perturbative $F_{\pi}^{2}$ expression starting at one-loop but zeroth $g^{2}$ order.

The discussion of the previous section on the analyticity domain of Eq. (3.5) with respect to $\Delta \gamma_{1}$ is identical, since the branch cuts are determined by the very same relation defining $F$, Eq. (2.34). One can thus proceed to a numerical optimization with respect to the RS dependence, along the same lines as in Sec. II E. The only difference is the Padé approximant form to be used: From Eq. (3.5), for $m^{\prime \prime \prime} \rightarrow 0$ the simple pole behavior is now given by the second perturbative term, with coefficient $\alpha_{\pi}^{\prime}$, while the first perturbative term, 1 , gives a vanishing contribution for $m^{\prime \prime \prime} \rightarrow 0$. A Padé approximant taking into account these properties and using the full information as contained in $\alpha_{\pi}^{\prime}, \beta_{\pi}^{\prime}$ is

$$
\left(1+\frac{\alpha_{\pi}^{\prime}(a)}{F^{\prime}}+\frac{\beta_{\pi}^{\prime}(a)}{\left(F^{\prime}\right)^{2}}+\cdots\right) \simeq \frac{1+\lambda /\left(F^{\prime}\right)^{2}}{1+\rho / F^{\prime}} \equiv P_{2,1}\left(F^{\prime}\right),
$$

which gives the result 


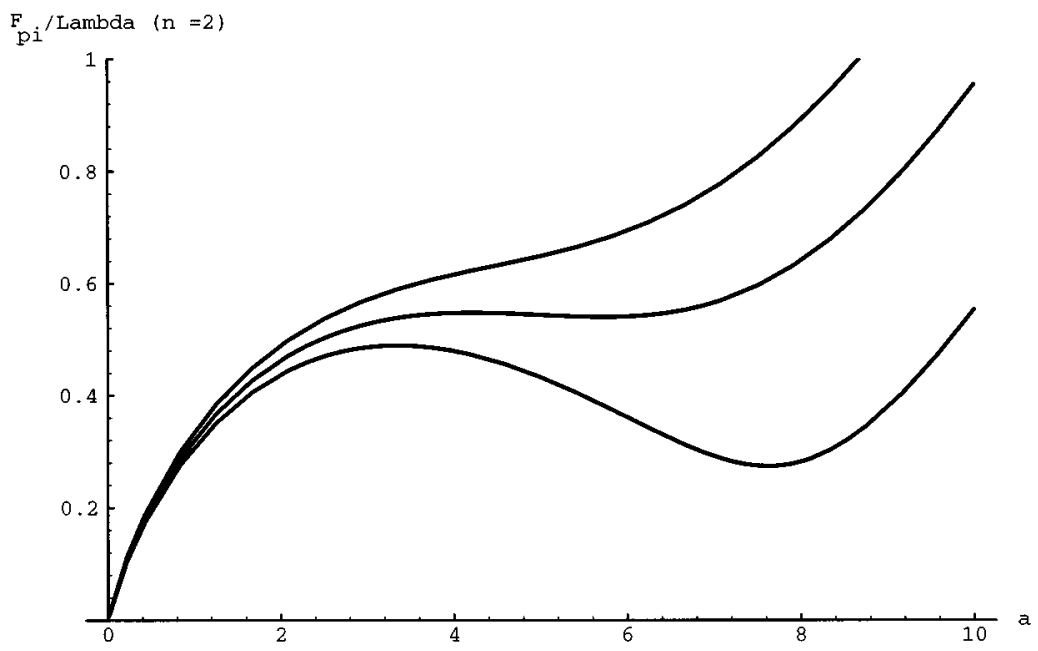

$$
\begin{aligned}
F_{\pi, \text { Padè }}^{2}(\cdots)= & \Lambda_{\overline{\mathrm{MS}}}(2 C)^{-2 C} a^{2} \exp \left\{\frac{2 A_{1}}{2 b_{0}}\right\} \\
& \times 2 b_{0} \delta_{\pi}\left[\alpha_{\pi}^{\prime}(a, \cdots)-\frac{\beta_{\pi}^{\prime}(a, \cdots)}{\alpha_{\pi}^{\prime}(a, \cdots)}\right],
\end{aligned}
$$

where the dots denote the RS dependence. The region around the optimum of Eq. (3.10) with respect to the RS parameters is illustrated in Fig. 2. In that case again we found a nontrivial flattest extrema minimizing the curvature (plateau), corresponding, e.g., for the $n_{f}=2$ case to $a_{\mathrm{opt}} \simeq 4.21, B_{1, \mathrm{opt}}$ $\simeq-0.017$. The optimum values are

$$
F_{\pi}^{\text {Padé }}(\mathrm{opt}) \simeq 0.55 \Lambda_{\overline{\mathrm{MS}}}(2)\left[0.59 \Lambda_{\overline{\mathrm{MS}}}(3)\right],
$$

for $n_{f}=2$ (3), respectively. This is also summarized in Table I. From Eq. (3.11), with $F_{\pi} \simeq 92 \mathrm{MeV}$ (and neglecting any explicit symmetry breaking effects due to the nonzero $u, d, s$ masses), one thus obtains $\Lambda_{\overline{\mathrm{MS}}}(2) \simeq 167 \mathrm{MeV}$ and $\Lambda_{\overline{\mathrm{MS}}}(3)$ $\simeq 156 \mathrm{MeV}$. Note that these relatively low values are more in agreement with earlier estimates of $\Lambda_{\overline{\mathrm{MS}}}[35,58]$ than with the recent experimental measurements of $\alpha_{S}\left(M_{Z}\right)$ at LEP [59], the latter giving a larger $\Lambda_{\overline{\mathrm{MS}}}(3)$ if evolving from the $M_{Z}$ scale down to very low $Q^{2}$ with naive perturbation theory. This does not in principle exclude our results, due to the still large uncertainties in $\Lambda_{\overline{\mathrm{MS}}}(2)$ and $\Lambda_{\overline{\mathrm{MS}}}(3)$ at very low energies. Moreover, as pointed out in Ref. [60], it could always be that new physics contribution, e.g., supersymmetry, affects the running of $\alpha_{S}$ between the $M_{Z}$ scale and low energy.

Finally, like in the mass case, we also give for comparison the (a priori ambiguous) corresponding value of Eq. (3.10) in the original $\overline{\mathrm{MS}}$ scheme:

$$
F_{\pi}^{\text {Padé }}(\overline{\mathrm{MS}}) \simeq 0.40 \Lambda_{\overline{\mathrm{MS}}}(2)\left[0.64 \Lambda_{\overline{\mathrm{MS}}}(3)\right] .
$$

$$
\text { IV. }\langle\bar{q} q\rangle
$$

\section{A. Renormalization-group-invariant $m\langle\bar{q} q\rangle$ Ansatz}

The (bare) quark-antiquark condensate is most conveniently defined as

FIG. 2. Dynamical $F_{\pi} / \Lambda_{\overline{\mathrm{MS}}}$ contribution from expression (3.10) with $n_{f}=2$ versus the scale parameter $a$, for different values of $B_{1}$ near the optimal (plateau) region. The exact plateau corresponds to $B_{1}=-0.0173 . \Delta \gamma_{1}$ is fixed to 0.00437 , such that $\operatorname{Re}\left[y_{\text {cut }}\right] \simeq 0$.

$$
\langle\bar{q} q\rangle \equiv-\frac{i}{n_{f}} \lim _{x \rightarrow 0} \operatorname{Tr} S(x),
$$

where $S(x)=i\langle 0|T \bar{q}(0) q(x)| 0\rangle$ is the quark propagator. ${ }^{25}$ While the above definition (4.1) is gauge invariant, as is well known $\langle\bar{q} q\rangle$ is not separately RG invariant, but $m\langle\bar{q} q\rangle$ is [56], which is thus the appropriate quantity to consider when applying the formalism developed in Sec. III A. Once subtracting the divergences remaining after mass and coupling renormalization, by following a construction similar to what was done for $F_{\pi}$, we obtain a finite Ansatz for $\bar{m}\langle\bar{q} q\rangle$. An awkward situation is that $\langle\bar{q} q\rangle$ cannot be directly accessed, and has to be extracted from tiny explicit symmetry breaking effects due to $m \neq 0$. This is of course a well-known problem, not specific to our construction. We shall discuss below in Sec. IV B a possible way of extracting a $\langle\bar{q} q\rangle(\mu)$ value from our construction.

The perturbative expansion up to two-loop order for $\bar{m}\langle\bar{q} q\rangle$ for $\bar{m} \neq 0$, calculated first in [62] and independently by us, is given explicitly in Appendix C. Note that the threeloop order is not known, which is rather unfortunate since, according to the derivation in Sec. III A, it implies that one only knows unambiguously the first coefficient of $1 / F$ in the $m\langle\bar{q} q\rangle$ Ansatz. To nevertheless give a more accurate estimate, following the usage in such a case, we take into account in our calculations the known RG dependence at $\mathcal{O}\left(1 / F^{2}\right) .{ }^{26}$ Again, one assumes that the optimization with respect to the RS parameters $a, \Delta \gamma_{1}$, etc. [whose exact dependence at $\mathcal{O}\left(1 / F^{2}\right)$ is also known], partly takes into ac-

\footnotetext{
${ }^{25} \mathrm{An}$ equivalent definition is from the derivative with respect to $m_{0}$ of the vacuum energy. Note that in the $m \rightarrow 0$ limit, there is an arbitrary phase in the definition (4.1): The sign of $\langle\bar{q} q\rangle$ is thus fixed a posteriori, by requiring that for real $m>0,\langle\bar{q} q\rangle \leqslant 0$, to be consistent, e.g., with the familiar Gell-Mann-Oakes-Renner [61] relation. In what follows we shall designate by $\langle\bar{q} q\rangle$ only the magnitude of the quark condensate.

${ }^{26}$ This allows at least some comparison with the true first order estimates, defined as the expressions obtained in the limit $b_{1}=\gamma_{1}$ $=0$.
} 
count this ignorance of higher order terms.

The resulting expression, including the full RS dependence, reads

$$
\begin{aligned}
\frac{\bar{m}\langle\bar{q} q\rangle}{\Lambda_{\overline{\mathrm{MS}}}^{4}}= & \exp \left[\frac{4 A_{1}}{2 b_{0}}\right] 2 b_{0} \frac{2^{-4 C} a^{4}}{2 i \pi} \oint \frac{d y}{y} y^{4} e^{y / m^{\prime \prime \prime}} \\
& \times \frac{1}{F^{\prime 4 A^{\prime}-1}\left[C+F^{\prime}\right]^{4 B^{\prime}}} \\
& \times \delta_{\langle\bar{q} q\rangle}\left(1+\frac{\alpha_{\langle\bar{q} q\rangle}^{\prime}(a)}{F^{\prime}}+\frac{\beta_{\langle\bar{q} q\rangle}^{\prime}(a)}{F^{\prime 2}}+\cdots\right),
\end{aligned}
$$

with

$$
\begin{aligned}
\delta_{\langle\bar{q} q\rangle} & =\frac{N_{c}}{4 \pi^{2}} \frac{1}{2 \gamma_{0}-b_{0}}, \\
\alpha_{\langle\bar{q} q\rangle}^{\prime}\left(a, \Delta \gamma_{1}\right)= & \frac{1}{2 b_{0}}\left[\frac{4}{3}\left(2 \gamma_{0}-b_{0}\right)-\pi^{2}\left(2 \gamma_{1}^{\prime}-b_{1}\right)\right. \\
& \left.-2\left(b_{0}-2 \gamma_{0}\right) \ln a\right]
\end{aligned}
$$

and

$$
\begin{aligned}
\beta_{\langle\bar{q} q\rangle}^{\prime}\left(a, \Delta \gamma_{1}, B_{1}\right)= & \frac{1}{\left(2 b_{0}\right)^{2}}\left[f_{\langle\bar{q} q\rangle}^{(2)}\left(\Delta \gamma_{1}\right)+4 \gamma_{0}\left(2 \gamma_{0}-b_{0}\right)\right. \\
& \left.\times \ln ^{2} a-10 \frac{\left(2 \gamma_{0}-b_{0}\right)}{6 \pi^{2}} \ln a\right] \\
& -\frac{4 B_{1}}{2 b_{0}} \alpha_{\langle\bar{q} q\rangle}\left(a, \Delta \gamma_{1}\right)+\frac{B_{1}}{\left(2 b_{0}\right)^{2}} \\
& \times\left[\frac{10}{3}\left(2 \gamma_{0}-b_{0}\right)-4 \pi^{2}\left(2 \gamma_{1}-b_{1}\right)\right],
\end{aligned}
$$

where $f_{\langle\bar{q} q\rangle}^{(2)}$ is given in Appendix C.

\section{B. Explicit chiral symmetry breaking corrections}

Clearly what we are really interested in is the value of the condensate in the exact chiral limit, $\bar{m} \rightarrow 0$. Closely related to the latter problem, it is desirable to obtain a connection between our construction and the familiar Gell-Mann-OakesRenner (GOR) relation [61], e.g., in the $S U(2)$ case,

$$
-\left(m_{u}+m_{d}\right)\langle\bar{u} u\rangle \simeq F_{\pi}^{2} m_{\pi}^{2}+\mathcal{O}\left(m_{u, d}^{2}\right),
$$

where $\langle\bar{u} u\rangle=\langle\bar{d} d\rangle$ for exact $S U(2)$ isospin symmetry, $F_{\pi}$ $\simeq 92 \mathrm{MeV}$, and $m_{\pi}$ are the pion decay constant and mass, respectively.

By definition in Eq. (4.6) $m_{u, d}$ are the current masses, which break the chiral $S U(2)_{L} \times S U(2)_{R}$ symmetry explicitly. In contrast, as amply discussed, in Eq. (4.2) $m^{\prime \prime \prime}$ is an arbitrary parameter, destined to reach the chiral limit $m^{\prime \prime \prime} \rightarrow 0$. Accordingly, $\bar{m} \rightarrow 0$ for $m^{\prime \prime \prime} \rightarrow 0$, so that one presumably expects only to recover the result $\bar{m}\langle\bar{q} q\rangle \rightarrow 0$ for $m^{\prime \prime \prime} \rightarrow 0$. This is actually the case: Although Eq. (4.2) may potentially give a nontrivial result in the chiral limit, typically the simple pole residue $\left[\simeq 2 b_{0}(2 C)^{-C} \delta_{\langle\bar{q} q\rangle} \alpha_{\langle\bar{q} q\rangle}(a)\right.$, upon neglecting higher order corrections], once we require extrema of this expression with respect to RS changes (using for the $m^{\prime \prime \prime} \rightarrow 0$ limit a Padé approximant similar to the one for $F_{\pi}$ ), we do not find nonzero extrema. Therefore, such a result is not conclusive regarding the actual value of $\langle\bar{q} q\rangle(\bar{\mu})$, although it may be considered a consistency cross-check of our formalism.

Now, one possible way of extracting $\langle\bar{q} q\rangle(\mu)$ from the Ansatz (4.2) is the following. Let us introduce a small explicit chiral symmetry breaking mass term,

$$
-m_{0, \operatorname{expt}} \bar{q}_{i} q_{i}
$$

to the basic Lagrangian (2.3). After renormalization, and carefully following the different steps as indicated in Sec. II and Appendix A, one can show that it amounts simply to the following substitution into the integrand of the different previous Ansätze:

$$
y \rightarrow y+m_{\text {expt }}^{\prime \prime}
$$

except in the factor $(d y / y) e^{y / m^{\prime \prime}}$, which remains unaffected, and where $m_{\text {expt }}^{\prime \prime}$ is related to $\bar{m}_{\text {expt }}$ in exactly the same way as $m^{\prime \prime}$ is related to $\bar{m}$; see Eq. (2.33). Substituting Eq. (4.8) into Eq. (4.2), one can now expand the right-hand side of Eq. (4.2), first for small $\left(y+m_{\text {expt }}^{\prime \prime}\right)$, and take in the resulting expression the true chiral limit, $y \rightarrow 0$. By finally subtracting $\bar{m}\langle\bar{q} q\rangle$ on both sides it gives an expression which depends on $m_{\text {expt }}^{\prime \prime}$ only, in the limit $m^{\prime \prime} \rightarrow 0$. More precisely one obtains after simple algebra

$$
\begin{aligned}
\bar{m}_{\text {expt }}\langle\bar{q} q\rangle \equiv & \lim _{m^{\prime \prime} \rightarrow 0}\left\{\left(\bar{m}+\bar{m}_{\text {expt }}\right)\langle\bar{q} q\rangle-\bar{m}\langle\bar{q} q\rangle\right\} \\
= & \Lambda_{\overline{\mathrm{MS}}}^{4}(2 C)^{-4 C} \delta_{\langle\bar{q} q\rangle} 2 b_{0} C^{(C-B) / A} \\
& \times \exp \left[\frac{4 A_{1}}{2 b_{0}}\right]\left(m_{\text {expt }}^{\prime \prime}\right)^{1 / A}+\mathcal{O}\left(m_{\text {expt }}^{\prime \prime}\right)^{2 / A},
\end{aligned}
$$

in terms of the quantities already defined after Eq. (4.2), including the RS dependence. ${ }^{27}$

Using the relation between $m_{\text {expt }}^{\prime \prime}$ and $\bar{m}_{\text {expt }}$ in Eq. (2.33), one can now cancel $\bar{m}_{\text {expt }}$ on both sides of Eq. (4.9) and

\footnotetext{
${ }^{27}$ The $(1 / A) \neq 1$ power of $m_{\text {expt }}^{\prime \prime} \propto \bar{m} / \Lambda_{\overline{M S}}$ in the third line of Eq. (4.9) may seem awkward at first. It is consistent, however, with the known perturbative behavior of $\langle\bar{q} q\rangle(\mu)$ : From Eq. (4.9), $\langle\bar{q} q\rangle(\mu) \simeq m^{2 b_{0} / \gamma_{0}-1} \ln \left(\mu / \Lambda_{\overline{\mathrm{MS}}}\right)$ for $\mu \gg \Lambda_{\overline{\mathrm{MS}}}$, so that since $m$ $\simeq\left[\ln \left(\mu / \Lambda_{\overline{\mathrm{MS}}}\right)\right]^{-\gamma_{0} / 2 b_{0}}$ one recovers $\langle\bar{q} q\rangle(\mu) \simeq\left[\ln \left(\mu / \Lambda_{\overline{\mathrm{MS}}}\right)\right]^{\gamma_{0} / 2 b_{0}}$ asymptotically; i.e., the condensate runs as inversely as the mass, as it should.
} 
extract a value of $\langle\bar{q} q\rangle$. Note that the dependence upon $\bar{m}_{\text {expt }} \equiv m_{\text {expt }}(\bar{\mu})$ does not completely cancel out, since $A\left(\Delta \gamma_{1}\right) \neq 1$ in a general RS. Indeed in Eq. (4.9) there is an additional implicit dependence on the scheme, through the relation between $m_{\text {expt }}^{\prime \prime}$ and $\bar{m}_{\text {expt }}$ in Eq. (2.33): This is expected, otherwise since $m^{\prime \prime} \propto \bar{m} / \Lambda_{\overline{\mathrm{MS}}}$, one would obtain $\langle\bar{q} q\rangle \propto$ pure number $\times \Lambda_{\overline{\mathrm{MS}}}^{3}$, which we know cannot be the case, due to the inherent $\mu$ dependence of $\langle\bar{q} q\rangle$. Consequently, in contrast with the dynamical mass and $F_{\pi}$, it would not make much sense to invoke the PMS with respect to the scale dependence $a$. Therefore, we shall fix Eq. (4.9) to its $\overline{\mathrm{MS}}$ scheme value (i.e., $a=1, \Delta \gamma_{1}=B_{1}=0$ ), which indeed makes comparison with other works easier, since the quark condensate is generally given in the $\overline{\mathrm{MS}}$ scheme (and typically at $\bar{\mu}=1 \mathrm{GeV}$ ) [35,63]. Although we do not try thus to numerically optimize expression (4.9), one should remember that the Ansatz (4.2) is assumed to be already optimal with respect to the variational mass parameter $m^{\prime \prime}$. Indeed, the only remaining contribution from Eq. (4.2) in Eq. (4.9) at first order in $m_{\text {expt }}^{\prime \prime}$ is the simple pole, but with residue given by the RS-independent zeroth order terms, as a consequence of the $F^{-1}$ factor in Eq. (4.2). Accordingly, potentially nontrivial extrema, which may have appeared from the RS dependence at the next orders (as was the case for $M_{q}$ and $F_{\pi}$ ), are washed out. Taking into account the next order $m_{\text {expt }}^{\prime \prime}$ terms in Eq. (4.9) one may eventually obtain nontrivial extrema, but those small corrections are not expected to drastically change the results given below.

A more serious trouble when fixing the RS to $\overline{\mathrm{MS}}$ is that the $\overline{\mathrm{MS}}$ scheme value $\Delta \gamma_{1}=0$ is such that the extra singularities occur at $\operatorname{Re}[y]>0$, therefore rendering the expansion around the origin $y \simeq 0$ a priori completely ambiguous, as explained in Sec. II. However, in Eq. (4.9), $y$ and $m_{\text {expt }}^{\prime \prime}$ are by definition independent parameters, and so it turns out that the extra singularities, as given by the zeros of $d y / d F$, are independent of $m_{\text {expt }}^{\prime \prime}$ (equivalently, because the extra singularities are due to the Jacobian of the $F \rightarrow y$ transformation, and the $d y e^{y / m^{\prime \prime}}$ term remains unaffected by $m_{\text {expt }}$ ). We thus assume that the corresponding ambiguities cancel out in the difference in expression (4.9). Although we were not able to prove this statement rigorously, at least it is easily checked that the $\bar{m}\langle\bar{q} q\rangle$ part in Eq. (4.9), which contains the singularities, do cancel in the first order expansion in $m_{\text {expt }}^{\prime \prime}$. Within this assumption, we thus obtain the $\overline{\mathrm{MS}}$ result

$$
\frac{\langle\bar{q} q\rangle \frac{1 / 3}{\mathrm{MS}}}{\Lambda_{\overline{\mathrm{MS}}}}(\bar{\mu}) \simeq 0.647\left[\frac{\hat{m}(\bar{\mu})}{\Lambda_{\overline{\mathrm{MS}}}(2)}\right]^{0.073}, \quad 0.614\left[\frac{\hat{m}(\bar{\mu})}{\Lambda_{\overline{\mathrm{MS}}}(3)}\right]^{0.018},
$$

where the $\mu$ dependence is only due to the remaining dependence on $\hat{m}(\bar{\mu}) \equiv 1 / 2\left(\bar{m}_{u}+\bar{m}_{d}\right) \quad$ or $\hat{m}(\bar{\mu}) \equiv 1 / 3\left(\bar{m}_{u}+\bar{m}_{d}\right.$ $\left.+\bar{m}_{s}\right)$, for $n_{f}=2$ or $n_{f}=3$, respectively. Accordingly to extract a $\langle\bar{q} q\rangle(\mu)$ value, we obviously have to use as input the explicit quark (current) masses $\hat{m}(\bar{\mu})$. Since the only available values of the light quark masses are extracted from a QCD sum rule determination of $\langle\bar{s} s\rangle$ [63], and using the $\hat{m}_{u, d} / \hat{m}_{s}$ mass ratio [64] (and moreover assuming the validity of the GOR relation), it is to be considered more as a consistency check than a truly independent prediction. Note, however, that Eq. (4.10) is only weakly sensitive to the explicit breaking masses $\hat{m}$, due to the small power coefficients involved.

Taking the latest results for the running masses at $1 \mathrm{GeV}$, as collected in Ref. [64], ${ }^{28}$

$$
\hat{m}(2) \equiv \frac{1}{2}\left(\bar{m}_{u}+\bar{m}_{d}\right) \simeq 7.2 \pm 2.3 \mathrm{MeV}
$$

and

$$
\hat{m}(3) \equiv \frac{1}{3}\left(\bar{m}_{u}+\bar{m}_{d}+\bar{m}_{s}\right) \simeq 63.1 \pm 9.1 \mathrm{MeV},
$$

for $n_{f}=2,3$, respectively, this gives

$$
\begin{gathered}
\langle\bar{q} q\rangle \frac{1 / 3}{\mathrm{MS}}(1 \mathrm{GeV}) \simeq(0.500-0.525) \Lambda_{\overline{\mathrm{MS}}}(2), \\
(0.602-0.606) \Lambda_{\overline{\mathrm{MS}}}(3)
\end{gathered}
$$

Alternatively, observing that in fact in Eq. (4.10) the power of $\hat{m} / \Lambda_{\overline{\mathrm{MS}}}$ only depends on the RS parameter $\Delta \gamma_{1}$, via the quantity $A$ defined in Eq. (2.35), there may be a way of extracting $\langle\bar{q} q\rangle(\mu)$ in the exact chiral limit, $\bar{m} \rightarrow 0$, by simply choosing a RS such that $A=1$ exactly. This occurs for $\Delta \gamma_{1}=31 /\left(288 \pi^{4}\right)$ and $\Delta \gamma_{1}=5 /\left(192 \pi^{4}\right)$ for $n_{f}=2$ and 3 , respectively, and gives

$$
\langle\bar{q} q\rangle_{\hat{m} \rightarrow 0}^{1 / 3}(1 \mathrm{GeV}) \simeq 0.52 \Lambda_{\overline{\mathrm{MS}}}(2), 0.58 \Lambda_{\overline{\mathrm{MS}}}(3) .
$$

Note that the results (4.13) and (4.14) are fairly consistent, given the errors in the quark mass $\hat{m}$ estimates and the presumed intrinsic error of our method. The not so good agreement for $n_{f}=3$ may be attributed to the fact that we have neglected the mass of the strange quark in our (chiral symmetric) estimate of $F_{\pi} / \Lambda_{\overline{\mathrm{MS}}}$ and $\langle\bar{q} q\rangle^{1 / 3} / \Lambda_{\overline{\mathrm{MS}}}$ (which is probably not a very good approximation, as indicated, e.g., from the fact that $F_{K} / F_{\pi} \simeq 1.23$, experimentally).

We thus obtain, within our approach, rather small $\langle\bar{q} q\rangle$ values. To make our results consistent with the sum rules calculations [35], we would need typically $\Lambda_{\overline{\mathrm{MS}}} \simeq 400 \mathrm{MeV}$, which contradicts the results in Sec. III for $F_{\pi} / \Lambda_{\overline{\mathrm{MS}}}$. [The former value of $\Lambda_{\overline{\mathrm{MS}}}$ is, however, more consistent with the experimental results [59] from LEP, $\alpha_{S}\left(M_{Z}\right)=0.117$ \pm 0.005$.

There are several ways in which our results may be interpreted. First, it is not excluded that our way of performing the small expansion in $m_{\text {expt }}$ in Eq. (4.9), although mathematically well defined, may simply not be a physically sensible way of proceeding. Our framework amply relies on the continuation to very small values of the arbitrary mass parameter $\mathrm{m}^{\prime \prime}$, and thus it may perhaps be only capable of

\footnotetext{
${ }^{28}$ In Eqs. (4.11), (4.12) the values with errors added linearly were simply obtained from the errors quoted in [64].
} 
determining the zero order of a chiral symmetry breaking expansion in a small mass. This is rather unfortunate for the quark condensate, which always appears as suppressed by an explicit mass in any physical relation. Besides, we anyhow consider the results in Eqs. (4.10), (4.14) on a less firm basis than the ones for $M_{q}$ and $F_{\pi}$ in Eqs. (2.48) and (3.11), due to the lack of PMS optimization [and due to the possible remaining ambiguities of Eq. (4.2) in the $\overline{\mathrm{MS}}$ scheme as well $\left.{ }^{29}\right]$.

\section{SUMMARY AND DISCUSSION}

Let us first summarize our main results.

We have shown that the variational expansion in arbitrary mass $\bar{m}$, as developed in the context of the GN model [8], can be formally extended to the QCD case. In particular we have seen how to reconcile the variational expansion with renormalization, and obtained a nontrivial, finite result in the chiral limit for the mass gap, by resumming the variational series expansion in $x$ with an appropriate contour integral.

Next, we have exhibited the link between the finite variational Ansatz and the RG solutions in a renormalized form, with the identification of specific fixed point boundary conditions. This allows us to generalize the construction in a most straightforward way to the next RG orders and to more complicated quantities as well, such as the relevant order parameters of CSB, $\langle\bar{q} q\rangle$, and $F_{\pi}$.

We have studied in some detail how the arbitrary RS dependence influences our different Ansätze and, in particular, how the extra singularities appearing in the QCD case can be moved away by an appropriate RS change at the second perturbative order. The resulting expressions in a generalized RS have been numerically optimized, within a certain approximation, due to the complexity of the complete optimization problem. The optimal values obtained for $M_{q} / \Lambda_{\overline{\mathrm{MS}}}$ and $F_{\pi} / \Lambda_{\overline{\mathrm{MS}}}$, summarized in Table I, are quite encouraging. Actually one should mention that the (unoptimized) corresponding values in the original $\overline{\mathrm{MS}}$ scheme, as given in Eqs. (2.50), (3.12), would equally well be in reasonable agreement with other estimates in the literature: Given the large present uncertainties in the values of $\Lambda_{\overline{\mathrm{MS}}}(2), \Lambda_{\overline{\mathrm{MS}}}(3)$ it is difficult to assert which results are closest to the largely unknown experimental values. However, the $\overline{\mathrm{MS}}$ values are $a$ priori ambiguous due to the extra singularities, as discussed in Sec. II D; moreover, according to the PMS principle adopted here, we consider on general grounds the optimized results as being on a firmer basis.

Finally, we have also introduced explicit chiral symmetry breaking mass terms in our framework, which plays an especially important role in extracting an estimate of $\langle\bar{q} q\rangle$. It should also be relevant to a more accurate evaluation of $F_{\pi} / \Lambda_{\overline{\mathrm{MS}}}$ and $M_{q} / \Lambda_{\overline{\mathrm{MS}}}$ in the $S U(3)$ case, where the explicit symmetry breaking due to the strange quark mass is presum-

\footnotetext{
${ }^{29}$ As previously we can also choose the $\operatorname{RS}$ where $\operatorname{Re}\left[y_{\text {cut }}\right]=0$ in Eqs. (4.2) and (4.9), which gives results in rough consistency with those in Eqs. (4.13), (4.14). However, because of the lack of RS invariance of $\langle\bar{q} q\rangle$, it is then more difficult to compare with the quoted results from other methods [35].
}

ably not negligible. We plan to study those explicit mass effects in a more quantitative way in a future work.

In Table I, we also give the results that are obtained when restricting the different Ansätze to the first order, i.e., taking the limit $b_{1}=0, \gamma_{1}=0$ and neglecting the $1 / F^{2}$ terms in the different expressions for $M_{2}^{P}, F_{\pi}$, and $m\langle\bar{q} q\rangle$, respectively. (In that case, the only arbitrariness is the renormalization scale, and the results were obtained from optimization with respect to $a$.) As well the "zero order" results, i.e., obtained by taking only the pure $R G$ dependence, are shown. Strictly speaking, those one-loop (and zero-loop) results are again $a$ priori ambiguous, being affected by the presence of extra singularities which cannot be moved away. We nevertheless found it useful to give them for completeness, as they were obtained from naively ignoring the ambiguities and taking the simple pole residues in the relevant Ansätze.

Now another problem is how to estimate the error of the method. As may be expected, the rigorous theorems in Ref. [30] cannot be applied to the present QCD framework. Admittedly, our final numerical results thus require a fair amount of mere "trust," which is the usual problem with the PMS, even indeed when applied to perturbative expressions. Note, however, that all our results are rigorously derived, as far as the pure RG behavior is concerned: It is only the purely perturbative non-RG corrections, $\mathcal{M}_{i} \neq 0$ in Eq. (2.42), which are treated according to the PMS. In other words we assume that it is legitimate to treat the remaining perturbative correction with a variant of the PMS. In the GN model case [8], the value of the curvature with respect to $a$ at the extremum gave a rather good qualitative idea of the error, but there is a priori no more definite relationship between the curvature at extrema points and the intrinsic error. Knowledge of the exact GN results allowed a comparison of different Padé approximant forms. As a partial qualitative cross-check, we have tried a similar comparison in the present case, and indeed found nontrivial optima (when those existed) of the same order of magnitude than as Eqs. (2.48), (3.11) with alternative Padé constructions similar to those explained in Ref. [8]. ${ }^{30}$ We do not find it sensible to make a systematic study in the QCD case since, as above discussed, it would not give much more of a quantitative idea on the intrinsic error of the method itself.

Concerning the relatively low $\langle\bar{q} q\rangle$ values, letting go the possibility that our method simply fails to extract an accurate estimate in the chiral limit, for the reasons discussed above, let us consider taking our $\langle\bar{q} q\rangle$ results at face value and discuss their consequences. In fact, the possibility of a marginally small (or even zero) quark condensate was recently raised in Refs. [51,52]. Clearly, $\langle\bar{q} q\rangle \neq 0$ is a sufficient but not necessary condition for spontaneous CSB. For instance, as mentioned in Sec. III, $F_{\pi}$ is an equally well-defined order parameter. In Ref. [51] the authors, moreover, emphasize that there are at present no clear experimental evidence for a realization of CSB through a large quark condensate. As stressed there, the familiar GOR relation is only first order in

\footnotetext{
${ }^{30}$ In the GN model analysis, we could not use a Padé approximant like in Eq. (2.47), which would give inconsistent results due to the fact that $b_{1}^{G N}<0$.
} 
the $\bar{m}_{\text {expt }}$ expansion, assuming the dominance of the $\langle\bar{q} q\rangle$ condensate, precisely. On the other hand, one may argue that there is some evidence, in particular from the spectral sum rules [35] or more recently from lattice simulations [5], of a low-energy QCD picture with a larger $\langle\bar{q} q\rangle$. On the theoretical side, giving up the dominance of the quark condensate gives a rather complicated framework to describe low-energy QCD: Although a generalization of chiral perturbation theory is perfectly possible [51], it loses a good part of its predictive power. Nevertheless, it is certainly not unreasonable to conclude that a small quark condensate is at present not excluded from the data.

Another possibility would be simply that the dominant contribution to quark condensation has a very different origin than the mechanism leading to $F_{\pi} \neq 0$ and dynamical quark masses $M_{q} \gg m_{q}$. As mentioned in the Introduction other nonperturbative effects associated with instantons, which are totally ignored here, are likely to play some role in the CSB dynamics. For instance, it was argued long ago [13], in some simplified picture of the QCD vacuum, that the instanton-anti-instanton interaction can give rise to effective (nonlocal) four-quark interactions of the Nambu-JonaLasinio (NJL-) type [2].

In any event, the present construction may be considered a definite estimate of the contributions to order parameters that are associated with the noninstantonic vacuum.

\section{ACKNOWLEDGMENTS}

I am indebted to André Neveu who first triggered my interest in the subject and for many useful discussions. I am grateful to Eduardo de Rafael for valuable criticisms and remarks at a earlier stage of this work, and also to Chris Arvanitis, Fredéric Geniet, Georges Grunberg, Heinrich Leutwyler, Gilbert Moultaka, Jan Stern, and Christoph Wetterich for helpful discussions.

\section{APPENDIX A: RENORMALIZATION GROUP MATERIAL}

We set here some definitions and normalization conventions for the renormalization group results used at different stages of our construction.

The homogeneous RG operator (i.e., giving zero when applied to a RG-invariant quantity) is taken as

$$
\mu \frac{d}{d \mu} \equiv \mu \frac{\partial}{\partial \mu}+\beta(g) \frac{\partial}{\partial g}-\gamma_{m}(g) m \frac{\partial}{\partial m},
$$

where the RG coefficients are given by

$$
\begin{gathered}
\beta(g) \equiv \mu \frac{d g}{d \mu}=-b_{0} g^{3}-b_{1} g^{5}-b_{2} g^{7}-\cdots, \\
\gamma_{m}(g) \equiv-\frac{\mu}{m} \frac{d m}{d \mu}=\gamma_{0} g^{2}+\gamma_{1} g^{4}+\gamma_{2} g^{6}+\cdots,
\end{gathered}
$$

in terms of the coupling constant $g$, in a minimal subtraction scheme. In QCD $\left[\alpha_{S} \equiv g^{2} /(4 \pi)\right]$ the coefficients $b_{i}$ and $\gamma_{i}$ are known up to three-loop order [46] and read explicitly $[41,42,46]$, in the $\overline{\mathrm{MS}}$-scheme,

$$
\begin{gathered}
b_{0}=\frac{1}{16 \pi^{2}}\left(11-\frac{2}{3} n_{f}\right), \\
b_{1}=\frac{1}{\left(16 \pi^{2}\right)^{2}}\left(102-\frac{38}{3} n_{f}\right), \\
b_{2}=\frac{1}{\left(16 \pi^{2}\right)^{3}}\left(\frac{2857}{2}-\frac{5033}{18} n_{f}+\frac{325}{54} n_{f}^{2}\right), \\
\gamma_{2}=\frac{1}{\left(16 \pi^{2}\right)^{3}}\left[\frac{1}{3}+\frac{404}{\left(16 \pi^{2}\right)^{2}}\left(\frac{40}{3}-\frac{4}{9} n_{f}\right),\right. \\
\left.\left.\zeta(3)-\frac{320}{27}\right) n_{f}-\frac{280}{81} n_{f}^{2}\right],
\end{gathered}
$$

where $n_{f}$ is the number of active quark flavors.

As is well known ${ }^{31}$ only the first two $b_{i}$ 's and $\gamma_{0}$ are RS independent. For a general perturbative change of scheme (restricted to second order which is sufficient for our purpose),

$$
\begin{gathered}
g^{2} \rightarrow g^{\prime 2}=g^{2}\left(1+A_{1} g^{2}+A_{2} g^{4}+\cdots\right), \\
m \rightarrow m^{\prime}=m\left(1+B_{1} g^{2}+B_{2} g^{4}+\cdots\right),
\end{gathered}
$$

the modification of the beta and gamma coefficients in the new (primed) scheme reads

$$
\begin{gathered}
b_{0}^{\prime}=b_{0} \\
b_{1}^{\prime}=b_{1} \\
b_{2}^{\prime}=b_{2}-A_{1} b_{1}+\left(A_{2}-A_{1}^{2}\right) b_{0}
\end{gathered}
$$

and

$$
\begin{gathered}
\gamma_{0}^{\prime}=\gamma_{0}, \\
\gamma_{1}^{\prime}=\gamma_{1}+2 b_{0} B_{1}-\gamma_{0} A_{1} \equiv \gamma_{1}+\Delta \gamma_{1} \\
\gamma_{2}^{\prime}=\gamma_{2}+2 b_{1} B_{1}+2 b_{0}\left(2 B_{2}-B_{1}^{2}\right)-2 A_{1} \gamma_{1}^{\prime}-\gamma_{0} A_{2} .
\end{gathered}
$$

Accordingly one can set $b_{2}^{\prime}=0$ and $\gamma_{2}^{\prime}=0$ by choosing appropriately $A_{2}$ and $B_{2}$ in Eqs. (A7), (A8), for arbitrary $B_{1}$ and $A_{1}$. These expressions are used in Secs. II-IV to construct generalized RS-dependent Ansätze, at the second perturbative order.

Next we define the leading log (LL), next-to-leading log (NLL), etc., formal series for the quantities we are interested in. Let us start with the mass: From purely dimensional considerations, we write, for the RG invariant physical (pole) mass,

\footnotetext{
${ }^{31}$ For a review of RG properties, see the excellent textbook by Collins [56].
} 


$$
M^{P}=\bar{m} \sum_{p=0}^{\infty} a_{p}\left(\frac{\bar{m}}{\bar{\mu}}\right) \bar{g}^{2 p}
$$

where

$$
a_{p}\left(\frac{\bar{m}}{\bar{\mu}}\right) \equiv \sum_{r=0}^{p} a_{p, r}\left[\ln \left(\frac{\bar{m}}{\bar{\mu}}\right)\right]^{p-r},
$$

$\bar{\mu}$ is the $\overline{\mathrm{MS}}$ scale, and $\bar{m} \equiv m(\bar{\mu})$ the Lagrangian mass. The $a_{p, 0},(p \geqslant 1)$ coefficients of $[\ln (\bar{m} / \bar{\mu})]^{p}$ define the LL terms, the $a_{p, 1}(p \geqslant 2)$ coefficients of $[\ln (\bar{m} / \bar{\mu})]^{p-1}$ define the NLL terms, etc. Accordingly the $a_{p p}$ coefficients are the nonlogarithmic perturbative terms, at order $p$. Considering now the equation obtained by applying Eq. (A1) to Eq. (A9), as giving the $\mu$ dependence of $M^{P}$ for fixed $\vec{g}^{2}$, one obtains formal series for the LL, NLL, etc. [56]:

The LL series:

$$
-p a_{p, 0}=\left[\gamma_{0}+2 b_{0}(p-1)\right] a_{p-1,0} \quad\left(p \geqslant 1, a_{0,0} \equiv 1\right) .
$$

The NLL series:

$$
\begin{aligned}
(1-p) a_{p, 1}= & {\left[\gamma_{0}+2 b_{0}(p-1)\right] a_{p-1,1} } \\
& +\left[\gamma_{1}+2 b_{1}(p-2)\right] a_{p-2,0}+\gamma_{0} \\
& \times(p-1) a_{p-1,0}(p \geqslant 2),
\end{aligned}
$$

and so on.

The universal (RS-independent) LL series can be easily resummed as

$$
M_{1}^{L L}=m\left(1+2 b_{0} g^{2} L\right)^{-\gamma_{0} / 2 b_{0}}
$$

[where $L \equiv \ln (\bar{m} / \bar{\mu})]$. In contrast the NLL, NNLL, etc. series are RS dependent, and not straightforward to resum explicitly. We have seen in Sec. II C how to find a resummation expression for the NLL series which can be checked to correctly reproduce the terms formally defined in Eq. (A12) to all orders.

For of a composite operator $\mathcal{O}^{n}$ of naive mass dimension $n$ and depending only on $m$ and $g$, as considered in Secs. III and IV for $F_{\pi}^{2}$ and $\bar{m}\langle\bar{q} q\rangle$, respectively, ${ }^{32}$ it is straightforward to generalize the above expressions in Eqs. (A11),(A12). One thus obtains similar LL and NLL series, which we do not display explicitly. It is easily checked that the perturbative expansion of the different Ansätze does reproduce the correct formal series.

\section{APPENDIX B: A CONTOUR INTEGRAL RESUMMING THE $x$ DEPENDENCE}

We review here the formalism originally introduced in $[7,8]$ to obtain a nontrivial result for the resummation of the perturbative series in $x$, in the limit $x \rightarrow 1$. It leads to the dynamical mass Ansatz in Eqs. (2.26), (2.31) and its gener-

\footnotetext{
${ }^{32} \mathrm{We}$ only consider, however, composite operators which can be defined as two-point functions.
}

alization for the composite operators in Secs. III, IV.

Consider the one-loop RG-invariant (bare) expression for the mass $M_{1}$ as given in Eq. (2.8):

$$
M_{1}=\frac{m_{0}}{\left[1-b_{0} \Gamma(\epsilon / 2)(4 \pi)^{\epsilon / 2} g_{0}^{2}\left(M_{1}\right)^{-\epsilon}\right]^{\gamma_{0} / 2 b_{0}}} .
$$

Performing the substitution

$$
m_{0} \rightarrow m_{0}(1-x), \quad g_{0}^{2} \rightarrow g_{0}^{2} x,
$$

provides a new quantity $M_{1}(x)$. To pick up the $x^{q}$ order term in $M_{1}(x) \equiv \sum_{q=0}^{\infty} a_{q} x^{q}$ (having in mind that we are actually interested in the limit $x \rightarrow 1$ ), a convenient trick is by contour integration:

$$
M_{1}^{(q)} \underset{(x \rightarrow 1)}{\rightarrow} \sum_{k=0}^{q} a_{k}=\frac{1}{2 i \pi} \oint d x\left(\frac{1}{x}+\cdots+\frac{1}{x^{q+1}}\right) M_{1}(x) .
$$

Now performing the sum in Eq. (B3) exhibits a $(1-x)^{-1}$ factor, canceling the $(1-x)$ from Eq. (B2). This results in the expression ${ }^{33}$

$$
M_{1}^{(q)} \underset{(x \rightarrow 1)}{\rightarrow} \frac{1}{2 i \pi} \oint d x x^{-(q+1)} m_{0}\left[f_{0}(x)\right]^{-\gamma_{0} / 2 b_{0}},
$$

where the contour is counterclockwise around the origin, and for convenience we defined the (recursive) function

$$
f_{0}(x) \equiv 1-b_{0} x g_{0}^{2} \Gamma\left[\frac{\epsilon}{2}\right] m_{0}^{-\epsilon}(1-x)^{-\epsilon}\left(f_{0}\right)^{\epsilon \gamma_{0} / 2 b_{0}},
$$

dictated from Eq. (B1). $f_{0}(x)$ has evidently a power series expansion in $x$, but, less obviously, also admits an expansion in $(1-x)$, as noted by inverting its defining relation (B5). This implies in particular that $x=1$ is an (isolated) pole of $M_{1}$.

Provided that no extra singularities lie in the way, one may distort the integration contour in Eq. (B4) to go around the cut lying along the real positive axis and starting at $x$ $=1$. Actually, one can go a step further and reach the $q \rightarrow \infty$ limit: After distorsion of the contour, only the vicinity of $x$ $=1$ survives for $q \rightarrow \infty$, which one can analyze by changing the variable to

$$
1-x \equiv \frac{v}{q}
$$

and rescaling $m_{0}$ by introducing $m_{0}=m_{0}^{\prime} q$, keeping $m_{0}^{\prime}$ fixed as $q$ goes to infinity. One finds in place of Eq. (B4),

$$
M_{1} \underset{(q \rightarrow \infty)}{=} \frac{1}{2 i \pi} \oint \frac{d v}{v} e^{v} \frac{v m_{0}^{\prime}}{f_{0}(v)^{\gamma_{0} / 2 b_{0}}},
$$

\footnotetext{
${ }^{33} \mathrm{In}$ Eq. (B4) there appeared in fact a factor of $1-x^{-(q+1)}$, from which only the last term contributes to the integral due to the analyticity of $f_{0}(x)$ defined in Eq. (B5).
} 
where now $f_{0}(v) \equiv 1-b_{0} g_{0}^{2} \Gamma[\epsilon / 2]\left(m_{0} v\right)^{-\epsilon}\left(f_{0}\right)^{\epsilon \gamma_{0} / 2 b_{0}}$. Once performing the renormalization via $m_{0}=\bar{m} Z_{m}, g_{0}^{2}$ $=\bar{\mu} \epsilon Z_{g} \bar{g}^{2}, M_{1}$ in Eq. (B7) is now finite to all orders:

$$
M_{1}=\frac{1}{2 i \pi} \oint d v e^{v} \frac{\bar{m}}{f^{\gamma_{0} / 2 b_{0}}},
$$

where the renormalized function

$$
f=1+2 b_{0} \bar{g}^{2} \ln \left[\left(\frac{\bar{m} v}{\bar{\mu}}\right) f^{-\left(\gamma_{0} / 2 b_{0}\right)}\right] .
$$

We have thus shown how to recover finite quantities with a nontrivial $x$ expansion. Equation (B8), however, only includes the one-loop RG dependence. To make the connection with the pole mass one should include the necessary nonlogarithmic perturbative corrections, already present, e.g., at the one-loop order in Eq. (2.6). This can be done without affecting the contour integration properties, except that the resulting expression of $M_{1}$ has a more complicated structure around $v \simeq 0$, which can be, however, systematically expanded around the origin in the way discussed in Sec. II B. Generalization of the previous construction to the next RG order is possible [8] although here we shall use in Sec. II C a more convenient construction directly in terms of renormalized quantities.

\section{APPENDIX C: PERTURBATIVE RESULTS}

In this section we collect for completeness the known perturbative expansions for the three quantities of interest, $M(\bar{m}), F_{\pi}^{2}(\bar{m})$, and $\bar{m}\langle\bar{q} q\rangle(\bar{m})$, respectively, in the $\overline{\mathrm{MS}}$ scheme. These determine, among other things, the nonlogarithmic perturbative corrections in our different Ansätze, as well as serve as a cross-check of the LL and NLL expansion properties.

\section{Quark mass}

The two-loop pole quark mass was calculated in Ref. [43], with exact dependence on the (current) quark masses running in the loops. It includes LL and NLL terms plus the nonlogarithmic perturbative corrections. As we consider only the case with $n_{f}$ equal mass quarks, the results of [43] take a simpler, entirely analytical form

$$
\begin{aligned}
\frac{M_{Q}}{\bar{m}}= & 1+\gamma_{0}\left(\frac{2}{3}-L\right) \bar{g}^{2}+\left[\gamma_{0}\left(\frac{\gamma_{0}}{2}+b_{0}\right) L^{2}\right. \\
& \left.+\left(\frac{\gamma_{0}^{2}}{3}-\frac{4}{3} \gamma_{0} b_{0}-\gamma_{1}\right) L+\frac{K}{\left(4 \pi^{2}\right)^{2}}\right] \bar{g}^{4}
\end{aligned}
$$

in terms of the (renormalized) current quark mass $\bar{m}$, with $L \equiv \ln (\bar{m} / \bar{\mu})$, and

$$
\begin{aligned}
K= & \frac{\pi^{2}}{9} \ln 2+\frac{7}{18} \pi^{2}-\frac{\zeta(3)}{6}+\frac{3673}{288}-\left(\frac{\pi^{2}}{18}+\frac{71}{144}\right) n_{f} \\
& +\frac{\left(\pi^{2}-3\right)}{8}\left(n_{f}-1\right)-\frac{2}{3} \gamma_{0}^{2}\left(4 \pi^{2}\right)^{2}
\end{aligned}
$$

for $n_{f}$ (equal mass) quarks [ $\zeta(x)$ is the Riemann zeta function]. We refer to [43] for the details of this calculation.

$$
\text { 2. } \boldsymbol{F}_{\pi}
$$

As discussed in Sec. III, the perturbative contributions to the pion decay constant $F_{\pi}$ may be obtained from the axialvector-axial-vector two-point correlator evaluated at $p^{2}=0$, where $p$ is the external momentum. But that is formally the very same quantity which appears as the (neutral) part of the $\rho$ parameter (at $p^{2}=0$ ) in electroweak theory [65], up to some trivial overall factors. The two-loop pure QCD corrections with exact quark mass dependence were first computed in [54] and recently the three-loop corrections in [55]. The only caution is to convert some of these results, given in the on-shell scheme, to the $\overline{\mathrm{MS}}$ scheme. ${ }^{34}$ More trivially, those expressions were calculated for a top quark mass $m_{t}$, so that in the present context we replace $m_{t} \rightarrow \bar{m}$, where $\bar{m}$ designates the current (equal) mass of the light quarks. One thus has, for $D=4-\epsilon$,

$$
\begin{aligned}
F_{\pi, 0}^{2}(\text { pert })= & -N_{c} \frac{\bar{m}^{2}}{2 \pi^{2}}\left\{-\frac{1}{\epsilon}+L+\frac{\bar{g}^{2}}{16 \pi^{2}}\left[\frac{8}{\epsilon^{2}}-\frac{10}{3 \epsilon}-8 L^{2}\right.\right. \\
& \left.-\frac{4}{3} L-\frac{1}{6}\right]+\left(\frac{\bar{g}^{2}}{16 \pi^{2}}\right)^{2}\left[-\frac{\frac{304}{3}-\frac{32}{9} n_{f}}{\epsilon^{3}}\right. \\
& +\frac{132-\frac{40}{9} n_{f}}{\epsilon^{2}}+\frac{-910 / 3+24 \zeta(3)+16 n_{f}}{9 \epsilon} \\
& \left.\left.+f_{\pi}^{30} L^{3}+f_{\pi}^{31} L^{2}+f_{\pi}^{32} L+f_{\pi}^{33}\right]\right\}
\end{aligned}
$$

where $L \equiv \ln (\bar{m} / \bar{\mu})$ and

$$
\begin{gathered}
f_{\pi}^{30}=\frac{304}{3}-\frac{32}{9} n_{f}, \\
f_{\pi}^{31}=-\frac{136}{3}+\frac{32}{9} n_{f}, \\
f_{\pi}^{32}=-8 \zeta(3)-\frac{149}{9}-\frac{10}{9} n_{f}, \\
f_{\pi}^{33}=\frac{16}{9}\left(\frac{51}{16}-36 \zeta(3)+27 \zeta(4)-6 B_{4}\right)+4\left(3+\frac{28}{3} \zeta(3)\right. \\
\left.-\frac{27}{2} \zeta(4)+3 B_{4}\right)+\frac{4}{3} n_{f}\left(-\frac{1}{12}+\frac{8}{3} \zeta(3)\right)
\end{gathered}
$$

\footnotetext{
${ }^{34}$ Results in the $\overline{\mathrm{MS}}$ scheme are also summarized in [55]. The expression in Eq. (C3) has been made consistent with our definition of $\epsilon$, which differs from the one in [54,55] by a factor of 2 .
} 


$$
-\frac{4}{3}[2+12 \zeta(3)]
$$

with

$$
B_{4}=16 \mathrm{Li}_{4}\left(\frac{1}{2}\right)+\frac{2}{3} \ln ^{4} 2-\frac{2}{3} \pi^{2} \ln ^{2} 2-\frac{13}{180} \pi^{4}=-1.7628 .
$$

As explicit, Eq. (C3) still contains divergences even after mass and coupling renormalization, and should be renormalized by and additional subtraction, according to the discussion in Sec. III. Using Eq. (3.4) one then obtains after some algebra the perturbative coefficients of $1 / F$ and $1 / F^{2}$, respectively, $\alpha_{\pi}$ and $\beta_{\pi}$ given in Eqs. (3.7),(3.8), where the twoloop term $f_{\pi}^{(2)}$ is given as

$$
\begin{aligned}
f_{\pi}^{(2)}(\overline{\mathrm{MS}})= & \frac{1}{1152 \pi^{4}\left(b_{0}+\gamma_{0}\right)}\left\{\left[455-24 n_{f}+24 \pi^{2}\left(\gamma_{0}+b_{0}\right)\right]\right. \\
& \times\left(\gamma_{0}-b_{0}\right)+1152 \pi^{4}\left(b_{2}-\gamma_{2}\right) \\
& +960 \pi^{4}\left(b_{0}-\gamma_{0}\right) \gamma_{1}+2304 \pi^{6} \gamma_{1}\left(\gamma_{1}-b_{1}\right) \\
& \left.+36\left(b_{0}-\gamma_{0}\right) \zeta(3)\right\} .
\end{aligned}
$$

\section{3. $\langle\bar{q} q\rangle$}

We have computed independently the perturbative expression of the quark-antiquark condensate $\langle\bar{q} q\rangle$, with a nonzero mass, to two-loop order. Our results fully agree with the expressions first calculated by the authors of Ref. [62]. With $D \equiv 4-\epsilon$, one obtains, in the $\overline{\mathrm{MS}}$ scheme,

$$
\begin{aligned}
m_{0}\langle\bar{q} q\rangle_{\text {pert }}^{0}= & N_{c} \frac{\bar{m}^{4}}{2 \pi^{2}}\left[\frac{1}{\epsilon}-L+\frac{1}{2}+\frac{\bar{g}^{2}}{\pi^{2}}\left(-\frac{1}{\epsilon^{2}}+\frac{1}{6 \epsilon}+L^{2}\right.\right. \\
& \left.\left.-\frac{5}{6} L+\frac{5}{12}\right)\right],
\end{aligned}
$$

where the index 0 stands for unrenormalized quantities. Like in the $F_{\pi}^{2}$ case, the expression in Eq. (C10) has to be further renormalized by a subtraction, which leads to the perturbative coefficients of $1 / F$ and $1 / F^{2}$ as given in Eqs. (4.4), (4.5), where the two-loop $f_{\langle q q\rangle}^{(2)}$ term $^{35}$ is given as

$$
\begin{aligned}
f_{\langle q q\rangle}^{(2)}(\overline{\mathrm{MS}})= & \frac{1}{6 \pi^{2}\left(b_{0}+2 \gamma_{0}\right)}\left[-5 b_{0}^{2}+6 \pi^{2}\left(b_{2}-2 \gamma_{2}\right)+20 \gamma_{0}^{2}\right. \\
& \left.+4 \pi^{2} b_{0} \gamma_{1}-12 \pi^{4} b_{1} \gamma_{1}-4 \gamma_{1}+24 \pi^{4} \gamma_{1}^{2}\right] .
\end{aligned}
$$

\footnotetext{
${ }^{35} \mathrm{We}$ stress again that $f_{\langle q q\rangle}^{(2)}$ is not the complete two-loop perturbative term: The latter would need the knowledge of the three-loop order $1 / \epsilon$ coefficient, which we arbitrarily put to zero here.
}

[1] J. Gasser and H. Leutwyler, Ann. Phys. (N.Y.) 158, 142 (1984); Nucl. Phys. B250, 465 (1985).

[2] Y. Nambu and G. Jona-Lasinio, Phys. Rev. 122, 345 (1961).

[3] See, e.g., J. Bijnens, C. Bruno, and E. de Rafael, Nucl. Phys. B390, 501 (1993); T. Hatsuda and T. Kunihiro, Phys. Rep. 247, 221 (1994), and Ref. [12] for a review of earlier works.

[4] M. Lüscher, R. Sommer, P. Weisz, and U. Wolf, Nucl. Phys. B413, 481 (1994).

[5] See, e.g., R. Gupta in Lattice 94, Proceedings of the International Symposium, Bielefeld, Germany, edited by F. Karsch et al. [Nucl. Phys. B (Proc. Suppl.) 42, 85 (1995)].

[6] B. Bellet, P. Garcia, and A. Neveu, Int. J. Mod. Phys. A 11, 5587 (1996); 11, 5607 (1996).

[7] C. Arvanitis, F. Geniet, and A. Neveu, hep-th/9506188.

[8] C. Arvanitis, F. Geniet, M. Iacomi, J.-L. Kneur, and A. Neveu, Int. J. Mod. Phys. A 12, 3307 (1997).

[9] See, e.g., S. Adler and R. Dashen, Current Algebras and Applications to Particle Physics (Benjamin/Cummings, New York, 1968).

[10] E. V. Shuryak, The QCD vacuum, Hadrons and the Superdense Matter (World Scientific, Singapore, 1988).

[11] "Dynamical Symmetry Breaking," Proceedings of the Nagoya Workshop, edited by T. Muta and K. Yamawaki, 1990 (unpublished).

[12] V. A. Miransky, Dynamical Symmetry Breaking in Quantum Field Theory (World Scientific, Singapore, 1993).

[13] C. G. Callan, R. Dashen, and D. J. Gross, Phys. Rev. D 17,
2717 (1978); E. V. Shuryak, Nucl. Phys. B203, 93 (1982); B203, 116 (1982); D. Dyakonov and V. Petrov, Sov. Phys. JETP 62, 204 (1985); 62, 431 (1985); Nucl. Phys. B272, 457 (1986).

[14] J. M. Cornwall, Phys. Rev. D 22, 1452 (1980).

[15] C. Arvanitis, F. Geniet, J.-L. Kneur, and A. Neveu, Phys. Lett. B 390, 385 (1997).

[16] P. M. Stevenson, Phys. Rev. D 23, 2916 (1981); Nucl. Phys. B203, 472 (1982) (and earlier references therein).

[17] W. E. Caswell, Ann. Phys. (N.Y.) 123, 153 (1979).

[18] I. G. Halliday and P. Suranyi, Phys. Lett. 85B, 421 (1979).

[19] J. Killinbeck, J. Phys. A 14, 1005 (1981).

[20] R. Seznec and J. Zinn-Justin, J. Math. Phys. 20, 1398 (1979).

[21] A. Okopinska, Phys. Rev. D 35, 1835 (1987).

[22] A. Duncan and M. Moshe, Phys. Lett. B 215, 352 (1988); H. F. Jones and M. Moshe, ibid. 234, 492 (1990).

[23] A. Neveu, in Recent Advances in Field Theroy, Proceedings of the Meeting, Annecy-le-vieux, France, 1990, edited by P. Bientruy et al. [Nucl. Phys. B (Proc. Suppl.) 18B, 242 (1990)].

[24] S. Gandhi, H. F. Jones, and M. Pinto, Nucl. Phys. B359, 429 (1991).

[25] H. Yamada, Z. Phys. C 59, 67 (1993).

[26] C. Bender et al., Phys. Rev. D 45, 1248 (1992) (and many earlier references therein); S. K. Gandhi and M. B. Pinto, ibid. 46, 2570 (1992).

[27] A. N. Sissakian, I. L. Solovtsov, and O. P. Solovtsova, Phys. Lett. B 321, 381 (1994). 
[28] G. Grunberg, Phys. Lett. 95B, 70 (1980); Phys. Rev. D 29, 2315 (1984).

[29] S. Brodsky, G. Lepage, and P. Mackenzie, Phys. Rev. D 28, 228 (1983).

[30] A. Duncan and H. F. Jones, Phys. Rev. D 47, 2560 (1993); C. M. Bender, A. Duncan, and H. F. Jones, ibid. 49, 4219 (1994); C. Arvanitis, H. F. Jones, and C. Parker, ibid. 52, 3704 (1995); R. Guida, K. Konishi, and H. Suzuki, Ann. Phys. (N.Y.) 241, 152 (1995); 249, 109 (1996).

[31] P. Forgacs, F. Niedermayer, and P. Weisz, Nucl. Phys. B367, 123 (1991); B367, 157 (1991).

[32] D. J. Gross and A. Neveu, Phys. Rev. D 10, 3235 (1974).

[33] U. Ellwanger and C. Wetterich, Nucl. Phys. B423, 137 (1994); D. Jungnickel and C. Wetterich, Phys. Rev. D 53, 5142 (1996).

[34] K. G. Wilson and J. G. Kogut, Phys. Rep. 12, 75 (1974).

[35] M. Shifman, A. Vainstein, and V. Zakharov, Nucl. Phys. B147, 385 (1979); B147, 488 (1979); B147, 519 (1979); L. Reinders, H. Rubinstein, and S. Yazaki, Phys. Rep. 127, 1 (1985); S. Narison, QCD Spectral Sum Rules, Lecture Series in Physics Vol. 26 (World Scientific, Singapore, 1989); Vacuum Structure and QCD Sum Rules, Current physics: sources and comments Vol. 10, edited by M. Shifman (North-Holland, Amsterdam, 1992).

[36] G. 't Hooft, Phys. Rev. Lett. 37, 8 (1976).

[37] R. Tarrach, Nucl. Phys. B183, 384 (1981).

[38] J. Breckenridge, M. Lavelle, and T. Steele, Z. Phys. C 65, 155 (1995).

[39] G. 't Hooft, in The Whys in Subnuclear Physics, Proceedings of the Erice Summer School, edited by A. Zichichi (Plenum, New York, 1977), p. 943; A. Mueller, in QCD Twenty Years Later, Aachen, 1992, edited by P. Zerwas and H. Kastrup (World Scientific, Singapore, 1992), and references therein.

[40] J.-L. Kneur, "Renormalons properties in QCD with non-zero quark masses" (in preparation).

[41] D. Gross and F. Wilczek, Phys. Rev. Lett. 30, 1323 (1973); Phys. Rev. D 8, 3633 (1973); H. D. Politzer, Phys. Rev. Lett. 30, 1346 (1973).

[42] W. Caswell, Phys. Rev. Lett. 33, 244 (1974); D. R. T. Jones, Nucl. Phys. B75, 531 (1974).

[43] N. Gray, D. J. Broadhurst, W. Grafe, and K. Schilcher, Z. Phys. C 48, 673 (1990).

[44] W. A. Bardeen, A. Buras, D. Duke, and T. Muta, Phys. Rev. D 18, 3998 (1978); A. Buras, Rev. Mod. Phys. 52, 199 (1980).
[45] W. J. Marciano, Phys. Rev. D 29, 580 (1984).

[46] O. Tarasov, A. Vladimirov, and A. Zharkov, Phys. Rev. B 93, 429 (1980).

[47] S. Peris and E. de Rafael, Phys. Rev. Lett. 387, 603 (1996).

[48] W. Celmaster and R. Gonsalves, Phys. Rev. D 20, 1420 (1979).

[49] M. A. Samuel, G. Li, and E. Steinfelds, Phys. Rev. E 51, 3911 (1995); M. A. Samuel, J. Ellis, and M. Karliner, Phys. Rev. Lett. 74, 4380 (1995).

[50] J. Bijnens, E. de Rafael, and H. Zheng, Z. Phys. C 62, 437 (1994).

[51] J. Stern, H. Sazdjan, and N. Fuchs, Phys. Rev. D 47, 3814 (1993); M. Knecht, H. Sazdjan, J. Stern, and N. Fuchs, Phys. Rev. B 313, 229 (1993).

[52] See, for a recent review, J. Stern, Talk given at 2nd DAPHNE Workshop, Frascati, 1995, hep-ph/9510318.

[53] H. Leutwyler, Phys. Rev. D 49, 3033 (1994).

[54] A. Djouadi, Nuovo Cimento A 100, 357 (1988); B. Kniehl, Nucl. Phys. B347, 86 (1990).

[55] L. Avdeev, J. Fleischer, S. Mikhailov, and O. Tarasov, Phys. Lett. B 336, 560 (1994); 349, 597 (E) (1995); K. Chetyrkin, J. Kühn, and M. Steinhauser, ibid. 351, 331 (1995).

[56] J. C. Collins, Renormalization (Cambridge University Press, Cambridge, England, 1984).

[57] H. Kluberg-Stern and J.-B. Zuber, Phys. Rev. D 12, 467 (1975).

[58] See, e.g., for a review D. Duke and R. Roberts, Phys. Rep. 120, 275 (1985).

[59] Particle Data Group, R. M. Barnett et al., Phys. Rev. D 50, 1173 (1994).

[60] M. Shifman, Mod. Phys. Lett. A 10, 605 (1995); Int. J. Mod. Phys. A 11, 3195 (1996); Phys. Rev. D 53, 404 (1996).

[61] M. Gell-Mann, R. Oakes, and B. Renner, Phys. Rev. 175, 2195 (1968).

[62] V. Spiridonov and K. Chetyrkin, Sov. J. Nucl. Phys. 47, 522 (1988).

[63] For the most recent analysis see J. Bijnens, J. Prades, and E. de Rafael, Phys. Lett. B 348, 226 (1995), and earlier references therein.

[64] H. Leutwyler, Talk given at Conference on Fundamental Interactions of Elementary Particles, Moscow, 1995, CERN-TH/ 96-25, hep-ph/9602255; Phys. Lett. B 378, 313 (1996).

[65] M. Veltman, Nucl. Phys. B123, 89 (1977). 